\title{
Energy-Efficient Transmission Strategy by Using Optimal Stopping Approach for Mobile Networks
}

\author{
Ying Peng, Gaocai Wang, ${ }^{2}$ and Nao Wang ${ }^{2}$ \\ ${ }^{1}$ College of Electrical Engineering, Guangxi University, Nanning 530004, China \\ ${ }^{2}$ School of Computer and Electronic Information, Guangxi University, Nanning 530004, China \\ Correspondence should be addressed to Gaocai Wang; wanggcgx@163.com
}

Received 18 September 2015; Revised 15 January 2016; Accepted 16 February 2016

Academic Editor: Qi Wang

Copyright (c) 2016 Ying Peng et al. This is an open access article distributed under the Creative Commons Attribution License, which permits unrestricted use, distribution, and reproduction in any medium, provided the original work is properly cited.

\begin{abstract}
In mobile networks, transmission energy consumption dominates the major part of network energy consumption. To reduce energy consumption for data transmission is an important topic for constructing green mobile networks. According to Shannon formula, when the transmission power is constant, the better the channel quality is, the greater the transmission rate is. Then, more data will be delivered in a given period. And energy consumption per bit data transmitted will be reduced. Because channel quality varies with time randomly, it is a good opportunity for decreasing energy consumption to deliver data in the best channel quality. However, data has delay demand. The sending terminal cannot wait for the best channel quality unlimitedly. Actually, sending terminal has to select an optimal time to deliver data before data exceeds delay. For this, this paper obtains the optimal transmission rate threshold at each detection slot time by using optimal stopping approach. Then, sending terminal determines whether current time is the optimal time through comparing current transmission rate with the corresponding rate threshold, thus realizing energy-efficient transmission strategy, so as to decrease average energy consumption per bit data transmitted.
\end{abstract}

\section{Introduction}

Widespread deployment of mobile networks, for example, mobile ad hoc networks and mobile social networks, and rapid development of data services have brought about exponential growth of wireless mobile terminals (MTs) and dramatic increase of energy consumption in mobile networks. However, the batteries of MTs offer very limited energy and lack the capacity for sustainable supply, especially in the case of inadequate network infrastructure or on the move. As energy consumption greatly affects mobile users, it is highly necessary to make efficient use of resources of mobile networks and reduce energy consumption of MTs for improving mobile users' satisfaction. It is an important and urgent subject to be solved for the construction of green mobile computing $[1,2]$.

In mobile networks environment, combined influence of multipath propagation, user on the move and channel fading, and so forth will bring about rapid fluctuations in capacity and quality of wireless channel with the change of time. If wireless networks always dynamically allocate resources to the channel in the best instantaneous state or MTs always choose the time of the best channel quality to transmit data, the utilization ratio of wireless network resources will be greatly increased and the performance of network is improved. MT delivers data efficiently using the feature of channel quality varying with time. This strategy is named as opportunistic scheduling [3].

There are two types of opportunistic scheduling, which include centralized opportunistic scheduling and distributed opportunistic scheduling. The former assumes the existence of a central scheduler, which can detect current status of all channels in the network and schedule operation and processing in a centralized manner. The latter, without knowing channel status of other devices, accesses and competes for channel in random mode and certain probability. After obtaining the channel through competition, it will either deliver data immediately if in good quality channel or give up and compete again if in bad quality channel, thus realizing full utilization of network resources in good quality channel.

The distributed opportunistic scheduling technique can take full advantage of device diversity of multiple users 
and the differences of channel in different time. In order to increase efficiency of the whole network, distributed opportunistic scheduling permits users to make decision after their temporary surveillance on channel. There is no such need of a complex control center and real time information of all channels' quality. In addition, this technique can decrease network energy consumption [4-8] and enhances network performance [9-12], for example, delivery ratio and throughput.

If the transmission power of sending terminal (ST) is given in wireless link, the greater the transmission rate is, the more data the ST can transmit within the same time period. Consequently, average energy consumption per unit data transmitted (AECPUDT) is smaller. However, transmission rate changes with the wireless channel quality fluctuations. If ST selects the time when channel is in good state to send data, the higher the transmission rate is, the lower the AECPUDT will be. Therefore, in order to minimize AECPUDT on the link, ST needs to survey channel condition timely and then chooses good channel quality time to transmit in accordance with present amount of data accumulated. ST chooses better channel quality for data delivery using the nature of channel quality changing with time, which is called distributed opportunistic scheduling [4-12]. In order to obtain an optimal energy efficiency time (i.e., AECPUDT is smallest) to deliver data, ST continuously surveys channel condition in distributed opportunistic scheduling. Therefore, the distributed opportunistic scheduling could be transformed into an optimal stopping rule strategy to be proved. In this strategy, the decision maker (ST) obtains the time of the minimal expected cost (average energy consumption) to stop observation, based on the continuous observation toward random variable (channel quality), and then takes special actions (transmitting data) to achieve the objective of minimal expected cost.

For the energy consumption problem research in [5], authors of that paper assumed that ST always had enough cumulative data and used the maximum transmission power at the maximum delay and then derived every transmission power threshold of each transmission slot time before the delay. Since current cumulative data quantity of ST is not taken into consideration of these thresholds, ST could neither achieve the optimal energy efficiency under different cumulative data quantity nor guarantee the data delivery ratio (i.e., the ratio of the amount of data transmitted to the total amount of data cumulated). In this paper, the authors take the amount of data cumulated in ST as related factor for selecting the optimal transmission time. In order to better derive the optimal stopping rule, it is assumed that the data generation rate of ST is given, which is consistent with reality needs. For example, when ST transmits some online data, the amount of data to be transmitted per unit time is identified. In addition, the rate of ST obtaining data to be forwarded is also assumed to be constant in $[6,7]$. Inspired by optimal stopping theory, the proposition of energy-efficient transmission strategy in this paper is based on the following ideas. When delay requirement and data generation rate on wireless link are given, the matter of distributed opportunistic scheduling about ST selecting optimal channel quality is turned into an optimal stopping problem. Then, the optimal threshold of transmission rate at every selection slot time is acquired based on the optimal stopping theory. Finally, the optimal rate time to transmit data is chosen, thus reducing the AECPUDT and increasing data delivery ratio.

The remaining part is arranged as follows. Related research work of other scholars is reviewed in Section 2. Section 3 describes system model and optimization problem. Then, the energy-efficient transmission strategy using optimal stopping approach is proposed in Section 4. Section 5 presents simulation results and analysis. Finally, Section 6 concludes this paper and prospects future work.

\section{Related Research Work}

At present, opportunistic scheduling based on time-varying wireless channel quality is widely researched and applied to mobile networks, such as mobile ad hoc networks and mobile social networks. These researches are mainly centered on selection of the optimal transmission time, aiming to improve network performance and energy efficiency of the three scenarios, namely, multiple devices and multiple channels, multiple devices and single channel, and single device and single channel. Researchers mainly focus on two optimization objectives, which are to improve energy efficiency [4-8, 1316] and to increase network throughput [9-12].

(1) For reducing network energy consumption, many scholars utilize method of choosing the optimal time of channel quality to transmit so as to reduce energy consumption of data transmission $[4-8,13]$. Others pay attention to energy consumption saving in the entire routing [14-16].

In order to decrease energy consumption for data transmission, the authors in $[4,5]$ studied how to choose optimal transmission time to improve energy efficiency in the environment of multiple devices and single channel as well as single device and single channel, respectively. The authors constructed an infinite horizon stopping problem based on optimal stopping theory in [4]. When the competing probability of multiple STs is given in homogeneous environment, the optimal threshold of transmission rate can be derived, which effectively optimized energy efficiency of network. They also proposed a heuristic method for heterogeneous scenario. But they did not consider the data transmission delay demand. The authors considered the case of data with the maximum transmission delay demand in [5]. They studied the transmission energy consumption optimization problem where channel quality varies with time. After obtaining the optimal threshold of power at each slot time using optimal stopping method, ST chose the optimal channel quality time to transmit data, thus energy consumption saved and delay guaranteed. According to their assumption, ST had adequate data to transmit all the time, which is an ideal case. In true applications, ST may have much more or much less data to deliver during the transmission period, while ST obtains excellent opportunities to transmit. The authors in [6] studied the cost optimization of that roadside unit transmitting data to passing-by vehicle in vehicle delay tolerant network. By introducing a function of transmission cost and a penalty cost for exceeding delay, they proved that the time when the queueing delay at roadside unit is above certain threshold is an optimal 
one for roadside unit to deliver data flow. The simulation results show that the optimal stopping theory can effectively save transmission cost of roadside unit. Furthermore, in [7] they studied this problem and gave a more complete theoretical derivation and experimental proof. We proposed an energy consumption optimization strategy for data transmission in [8] and formed preliminary ideas of utilizing optimal stopping approach to save energy. We present more perfect theoretical derivation and abundant experimental proof in this paper, which includes five points mainly. The first is the full explanation of opportunistic scheduling problem using optimal stopping theory. The second is the detailed related research work and the differences between ours and the work of others in [5]. The third is the correlation between this work and optimal stopping theory. The fourth is the detailed solution and performance analysis of the strategy. The fifth is the relationship of optimal energy efficiency and parameters and analysis of average scheduling period. The problem of choosing good channel with delay constraint in mobile networks is studied by authors of [13]. They utilized stochastic game to obtain the optimal power threshold for successful data transmission in opportunistic scheduling, which reduced the waste of energy caused by channel error and packet collision.

In addition, some others focused on saving energy in the opportunistic network, which is consumed by sending information from source to destination. Due to rapid fluctuation of channel condition, routing information estimated by average channel quality will become out of date, but opportunistic routing can avoid this case. Compared with the traditional way that data is delivered through a predefined end-to-end path, opportunistic routing enables data to be transmitted from source to destination without end-to-end path. For example, [14] proposed a method of cooperative communication for energy efficiency. The authors fully exploited the random change nature of wireless channel and enabled data packet to be transmitted through better path by high energy efficiency relay node. Consequently, energy consumption is reduced. In [15], they introduced the functions of computing end-to-end energy consumption for traditional routing and opportunistic routing. The authors utilized the technique of cross-layer information exchange to reduce energy consumption and designed energy-efficient routing algorithms based on Dijkstra algorithm. The simulation results show that energy-efficient opportunistic routing outperforms the traditional routing. We in [16] proposed the routing strategy of minimizing transmission energy consumption in mobile network, where transmission delay demand of data is considered. The routing strategy decreased network energy consumption effectively.

(2) For increasing network throughput of opportunistic network, researchers propose all kinds of schemes and algorithms to select a good channel for data transmission, so as to obtain more desirable transmission rate and increase data delivery ratio.

In order to improve network throughput in an ad hoc network where many links contend for the same channel by random access, two distributed opportunistic scheduling strategies from the network-centric and user-centric perspective are proposed in [9], respectively. In [10], the authors studied the improvement of network throughput with proportional fairness. In the scenario of multiple devices competing for the same channel, the researchers constructed block fading channel model with different channel detection slot time dependencies in [11]. Taking into account the impact of channel dependencies on transmission scheduling and system performance, they formulated optimal stopping problem of finite horizon and chose the effective decision time, then characterized the system performance by backward induction, and finally solved the problem by recursive algorithm and effectively enhanced throughput of the system. Considering secure and regular links coexisting in a mobile network, [12] designed a QoS-oriented distributed scheduling scheme based on optimal stopping theory to maximize the whole network throughput.

In summary, for the distributed opportunistic scheduling, it is a very important solution to obtain the optimal scheduling time [4-12] using the optimal stopping theory. In these researches including multiple devices competing to select multiple channels, multiple devices competing to select single channel, and single device selecting single channel, researchers constructed different kinds of optimal rule problem. They solved these problems to obtain the optimal transmission rate threshold [4, 8-11], the optimal power threshold [5], and the optimal transmission time [6, 7], respectively, so as to minimize energy consumption [4-8] or maximize network throughout [9-12]. We research the energy-efficient transmission strategy in this paper, when one device selects single channel. Differing from the hypothesis in [5] that ST has adequate data all the time, we assume that data is generated at a certain rate. In addition, the objectives of minimizing expected energy consumption and average energy consumption per unit time are studied in [5]. This paper considers the objective of minimizing AECPUDT, under the constraint condition of finishing transmitting all data accumulated. For this purpose, we construct the minimization problem of AECPUDT with transmission delay demand and the amount of data transmitted constraint. This minimization problem can be turned into a finite horizon optimal stopping rule problem, so as to acquire the optimal transmission rate thresholds. This paper includes the following two main contributions. (1) The transmission energy consumption optimization problem with transmission delay demand and the amount of data transmitted constraint in mobile network is studied using the characteristics of channel quality varying with time. The effect of the given data generation rate and transmission delay on the AECPUDT is analyzed. (2) We construct the finite horizon optimal stopping rule problem about the minimal AECPUDT under amount of data transmitted constraint. Then, the optimal threshold of transmission rate at every slot time is obtained, thus forming the energy-efficient transmission strategy using optimal stopping approach.

\section{Theoretical Background and Problem Description}

3.1. System Model. In our mobile networks model, we assume that MT accesses channel using Carrier Sense Multiple Access 


\begin{tabular}{|l|l|l|l|l|}
$\overbrace{0}^{D}$ & $D$ & $D$ & $D$ & $S$ \\
\hline
\end{tabular}

Find good quality channel; stop to transmit

\begin{tabular}{l|c|c|c|c|}
\hline$D$ & $D$ & $\cdots$ & $D$ & $S$ \\
\hline 0 & 1 & \multicolumn{4}{c|}{$D_{m} / T$} \\
Detect until & $D_{m}$; stop to transmit
\end{tabular}

$\begin{array}{ll}D: \text { detect channel } & t: \text { transmission period } \\ S: \text { stop to transmit data } & D_{m} \text { : maximum delay } \\ T \text { : detection cycle } & \end{array}$

FIgURE 1: A round of channel detection and data transmission.

with Collision Avoidance (CSMA/CA) protocol. When data needs to be transmitted between two MTs, after establishing the wireless link within transmission range, MT will choose good channel to transmit data immediately. The research purpose of this paper is to minimize the AECPUDT in wireless link under the demand of transmission delay. Meanwhile, the delivery ratio of data transmission must be guaranteed.

In mobile networks, we assume that time is divided into certain slot periods $T$ (s). The channel gain $g$ submits some probability distribution (such as Rayleigh model fading) and remains unchanged in period $T[4,5,9,11]$ on the wireless link construed by ST and a receiving terminal (RT). The data generation rate is $c(\mathrm{bit} / \mathrm{s})$. ST delivers the data to the RT within the transmission delay $D_{m}$ using transmission power $P(\mathrm{~W})$. To obtain real time information of channel quality, RT transmits a short signal to ST every period $T$ [5]. Then, ST estimates channel quality according to the signal's power. This consumes energy $E_{D}(J)$ for each detection. Each duration of detection signal is extremely short and far less than $T$. After the ST discovers channel in good condition, it will send data during period $t . P \cdot t(\mathrm{~J})$ is the energy consumption for data transmission and far bigger than $E_{D}$. Because the channel gain $g$ keeps constant in period $T, t \leq T$ is satisfied. This entire process from the starting of channel detection to the end of data transmission is called a round of channel detection and data transmission. Its process is given in Figure 1. In a round of detection, the total duration for detection is $n \cdot T$ (s), and the total energy consumption for detection is $n \cdot E_{D}(\mathrm{~J})$. Here, $n$ is the number of detection instances, which is counted from the end of previous round of data transmission. For the first round, the initial counting of $n$ is 0 . If transmission rate is $R$ (bit/s), ST can transmit $R \cdot t$ (bit) data in a round. If there is $R t<c(n T+t)$, ST does not transmit all cumulated data and the remaining data is $c(n T+t)-R t$ bits. If $R t \geq c(n T+t)$, ST actually transmits $c(n T+t)$ bits' data. Furthermore, when $R t>c(n T+t)$ is satisfied, ST wastes power due to being idle in part of duration $t$. Obviously, ST can increase the amount of data transmitted in given transmission duration by selecting the time of greater transmission rate, thus decreasing the AECPUDT and improving energy efficiency. Shannon formula is presented in the following expression:

$$
R=W \log _{2}\left(1+\frac{g \cdot P}{N_{0} \cdot W}\right)
$$

According to expression (1), transmission rate $R$ in the channel is associated with transmission power $P$, channel gain $g$, noise power spectral density $N_{0}$, and bandwidth $W$. When the values of $W, P$, and $N_{0}$ are certain, the value of $R$ is in positive proportion to the value of $g$. Therefore, to select the time of greater transmission rate, ST must capture the time of larger channel gain value or better channel quality. In order to acquire the optimal channel quality time to transmit data, ST needs to know channel quality timely. The optimal time to transmit is obtained using optimal stopping approach in this paper.

3.2. Optimal Stopping Theory. In order to maximize the expected payoff or minimize the expected cost, the decision maker observes the random variables sequentially in the optimal stopping theory and selects a proper time to take a given action [17]. The stopping rule is defined by two objects:

(1) A sequence of random variables $X_{1}, X_{2}, \ldots$, whose joint distribution is assumed to be known.

(2) A sequence of real-valued rewards or cost functions $y_{0}, y_{1}\left(x_{1}\right), y_{2}\left(x_{1}, x_{2}\right), \ldots, y_{\infty}\left(x_{1}, x_{2}, \ldots\right)$.

The associated stopping rule may be described as follows. After observing $X_{1}=x_{1}, X_{2}=x_{2}, \ldots, X_{n}=x_{n}(n=$ $1,2, \ldots)$, the decision maker may stop observation and accept the known reward or cost function $y_{n}\left(x_{1}, \ldots, x_{n}\right)$ or continue observing $X_{n+1}$. If decision maker chooses not to take any observation, he accepts the constant value $y_{0}$. If he has not stopped observing, he will receive $y_{\infty}\left(x_{1}, x_{2}, \ldots\right)$. This rule enables that the decision maker chooses the optimal time $N$ $(0 \leq N \leq \infty)$ to stop observation, so that the expected reward $E\left[Y_{N}\right]$ is maximal or the expected cost $E\left[Y_{N}\right]$ is minimal. Among them, $Y_{N}=y_{N}\left(x_{1}, \ldots, x_{N}\right)$ is a random cost or reward stopping at $N$. E[.] expresses the mathematical expected value. If $n \rightarrow \infty$, this problem is an infinite horizon optimal stopping problem, which could be calculated through optimality equation. But, in real applications, $n$ is not beyond some value $N_{m}$. Then, the problem becomes a finite horizon one and is a special case of infinite horizon. It could be computed using backward induction, which is calculated from the maximum value $N_{m}$ to the minimum value 0 , reversely. Currently, there are a lot of such problems of selecting the optimal time to take actions to maximize the expected reward or minimize the expected cost in the field of communication. Hence, the optimal stopping theory about how to select optimal time according to random variables sequentially observed is an effective tool for solving the mentioned problems.

3.3. Problem Description. In order to minimize AECPUDT and guarantee the delivery ratio, ST selects the optimal time to transmit data under the premise of transmission delay $D_{m}$ according to the results of continuous observation on channel quality. Hence, the minimization problem in this paper is a finite horizon optimal stopping problem.

In the environment of mobile networks, both the mobility of MT and environmental interference will have some effect on channel quality. The channel condition is uncertain. When the transmission power of ST is constant, the better 


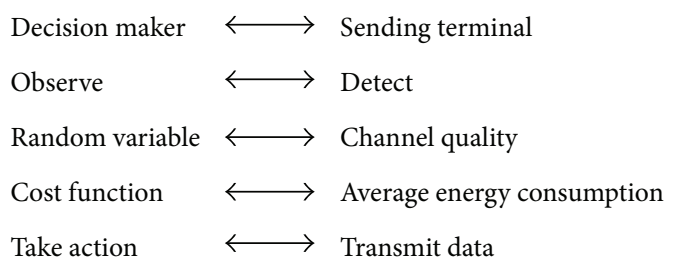

FIGURE 2: Optimal stopping problem elements in data transmission.

the channel quality is (i.e., the faster the transmission rate is), the more the amount of data transmitted in the same duration is. Hence, the AECPUDT is less. The result of the poor quality channel is contrary. At the same time, data to be delivered is generated in given rate and accumulates into ST. If the transmission interval is short, the amount of data accumulated is always less than the one that can be transmitted in transmission duration. Thus, ST wastes transmission power, which causes more AECPUDT. If the transmission interval is too long to transmit all cumulative data in transmission duration, part of data will be discarded due to exceeding the transmission delay.

Actually, MT must decide whether to send data at once or wait for next better moment to transmit data according to current channel quality and the amount of cumulative data. Obviously, due to the stochastic characteristics of link channel quality, ST must detect the channel quality, and then it selects the optimal time to stop detection and transmit data under the accumulative data quantity constraint and the delay demand, so as to minimize the AECPUDT. Therefore, we obtain the relevance between the optimal stopping theory and our optimal stopping problem about energy-efficient transmission strategy, which is shown in Figure 2.

\section{Energy-Efficient Transmission Strategy}

4.1. Construction of Average Energy Consumption Minimization Problem. We define variable sequence $X_{n}=\left\{\Delta T_{n}, R_{n}\right\}$ in the $n$th channel detection. Here, $\Delta T_{n}\left(\Delta T_{n}=T \cdot n\right)$ represents the duration of observation and $R_{n}$ means the transmission rate at $n$. ST must detect channel at least once. $N$ is assumed to be time index (time for short) when ST stops detection. The time at which ST must stop detection and deliver data is $M$, which is $\max \left\{n: \Delta T_{n} \leq D_{m}\right\}$. So $1 \leq n \leq N \leq M$ is satisfied. In a round of detection, the number of channel detection instances is $N$. Energy consumption for detection of every time is $E_{D}$. After the end of detection, ST transmits data in period $t$. The value of transmission energy consumption is $P \cdot t$. Hence, the total energy consumption in a round is presented in the following expression:

$$
E_{N}=N E_{D}+P t \text {. }
$$

If ST runs this given stopping rule in $Y$ rounds, there will be stopping time sequences $\left\{N_{1}, N_{2}, \ldots, N_{y}, \ldots, N_{Y}\right\}$, energy consumption sequences $\left\{E_{N_{1}}, E_{N_{2}}, \ldots, E_{N_{y}}, \ldots, E_{N_{Y}}\right\}$, and transmission rate sequences $\left\{R_{N_{1}}, R_{N_{2}}, \ldots, R_{N_{y}}, \ldots, R_{N_{Y}}\right\}$. Here, $N_{y}$ represents the stopping time in the $y$ th round. The initial value of stopping time in each round is 0 . With ST detecting channel once in the $y$ th round, the value of $N_{i}$ is increased by one. And there is $1 \leq N_{y} \leq M$. Hence, the total duration of the $y$ th round is sum of detection duration $\Delta T_{N y}\left(\Delta T_{N y}=T \cdot N_{y}\right)$ and transmission duration $t$. It is equal to $\Delta T_{N y}+t$. $E_{N_{y}}\left(E_{N_{y}}=N_{y} E_{D}+P t\right)$ is the total energy consumption of the $y$ th round stopping at $N_{y}$. At this moment, $c\left(\Delta T_{N y}+t\right)$ bits' data is accumulated and needed to be delivered. $R_{N y}$ is the transmission rate of the $y$ th round stopping at $N_{y} . L_{N y}$ is the value of data that could not be transmitted this time, which is given in the following expression:

$$
\begin{aligned}
L_{N y} & =\left(c\left(\Delta T_{N_{y}}+t\right)-R_{N y} t\right)^{+} \\
& = \begin{cases}c\left(\Delta T_{N_{y}}+t\right)-R_{N y} t, & c\left(\Delta T_{N_{y}}+t\right)>R_{N_{y}} t \\
0, & c\left(\Delta T_{N_{y}}+t\right) \leq R_{N_{y}} t .\end{cases}
\end{aligned}
$$

Hence, energy efficiency $\zeta$ of AECPUDT is defined in the following expression:

$$
\zeta=\frac{\sum_{y=1}^{Y} E_{N y}}{\sum_{y=1}^{Y}\left(c\left(\Delta T_{N y}+t\right)-L_{N y}\right)} .
$$

In accordance with the law of large numbers, expression (4) converges to $E\left[E_{N}\right] / E\left[c\left(\Delta T_{N}+t\right)-L_{N}\right]$, where $N$ is the time that ST stops detecting and $E[\cdot]$ represents the mathematical expected value. Thus, an optimal stopping rule problem is constructed, which selects stopping time $N$ to minimize $E\left[E_{N}\right] / E\left[c\left(\Delta T_{N}+t\right)-L_{N}\right]$. There is $1 \leq N \leq$ $M$. This rule comes from the transmission rate sequence $R_{N}$ and the total detection duration sequence $\Delta T_{N}$, which are detected by ST every period T. Meanwhile, energy consumption sequence $E_{N}$, accumulative data sequence $c\left(\Delta T_{N}+t\right)$, and data left undelivered sequence $L_{N}$ are generated. All these sequences values are measurable to be obtained.

When the transmission duration is fixed as $t$, the amount of data waiting for transmission is $c\left(\Delta T_{N}+t\right)$. If there is $R_{N} t<c\left(\Delta T_{N}+t\right)$, part of data is left undelivered in this round. On the other side, ST needs to send all data accumulated within duration $t$. Therefore, this optimization problem includes the constraint condition $\varphi$, which is $R_{N} t \geq$ $c\left(\Delta T_{N}+t\right)$. Consequently, the set of stopping time is given in the following expression:

$$
N^{+}=\left\{N: 1 \leq N \leq M, E\left[\Delta T_{N}\right] \leq D_{m}, \varphi\right\}
$$

So the minimization problem of AECPUDT with the amount of data transmitted constraint is described as follows:

$$
\begin{array}{ll}
\min _{N \in N^{+}} & \frac{E\left[N E_{D}+P t\right]}{E\left[c\left(\Delta T_{N}+t\right)-L_{N}\right]} \\
\text { s.t. } & E\left[R_{N} t\right] \geq E\left[c\left(\Delta T_{N}+t\right)\right] .
\end{array}
$$


4.2. Transformation of Optimal Stopping Problem about Average Energy Consumption Minimization. In this paper, it is necessary to derive optimal stopping rule and optimal energy efficiency (the minimum AECPUDT) of ST. In accordance with expression (4), optimal energy efficiency $\zeta^{*}$ is defined as follows:

$$
\zeta^{*}=\inf _{N \in N^{+}} \frac{E\left[N E_{D}+P t\right]}{E\left[c\left(\Delta T_{N}+t\right)-L_{N}\right]} .
$$

The above expression is turned into the equation in the following expression:

$$
\inf _{N \in N^{+}}\left(E\left[N E_{D}+P t\right]-\zeta^{*} E\left[c\left(\Delta T_{N}+t\right)-L_{N}\right]\right)=0 .
$$

The minimization problem of expression (8) is an optimal stopping problem related to $\zeta$ to minimize $E\left[Z_{N}\right]$. The following equation holds:

$$
Z_{N}=N E_{D}+P t-\zeta\left(c\left(\Delta T_{N}+t\right)-L_{N}\right) .
$$

Suppose, for every value of $\zeta$, a set of optimal time $N(\zeta) \in N^{+}$to minimize $E\left[Z_{N}\right]$ exists. Our objective is to acquire optimal stopping time $N^{*}=N\left(\zeta^{*}\right)$, so as to get the optimal energy efficiency $\zeta^{*}$. Hence, expression (10) is obtained. Consider

$$
N^{*}=\underset{N \in N^{+}}{\arg \inf } \frac{E\left[N E_{D}+P t\right]}{E\left[c\left(\Delta T_{N}+t\right)-L_{N}\right]} .
$$

Therefore, expression (6) is transformed as follows:

$$
\begin{array}{ll}
\min _{N \in N^{+}} & \left(E\left[N E_{D}+P t\right]-\zeta E\left[c\left(\Delta T_{N}+t\right)-L_{N}\right]\right) \\
\text { s.t. } & E\left[R_{N} t\right]-E\left[c\left(\Delta T_{N}+t\right)\right] \geq 0 .
\end{array}
$$

On the basis of the Lagrange duality theory, expression (11) is transformed into the following expression:

$$
\begin{aligned}
& \min _{N \in N^{+}} E\left[Y_{N}\right]=\min _{N \in N^{+}}\left(E\left[N E_{D}+P t\right]\right. \\
& \quad-\zeta E\left[c\left(\Delta T_{N}+t\right)-L_{N}\right] \\
& \left.\quad+\lambda\left(E\left[c\left(\Delta T_{N}+t\right)\right]-E\left[R_{N} t\right]\right)\right) .
\end{aligned}
$$

Here, $\lambda \geq 0$ is a Lagrange multiplier.

Remark 1. In expression (12), the following expression can be gotten:

$$
\begin{array}{ll}
\lambda=0, & E\left[c\left(\Delta T_{N}+t\right)\right] \leq E\left[R_{N} t\right], \\
\lambda>0, & E\left[c\left(\Delta T_{N}+t\right)\right]>E\left[R_{N} t\right] .
\end{array}
$$

Hence, we have the following expression:

$$
\lambda\left(E\left[c\left(\Delta T_{N}+t\right)\right]-E\left[R_{N} t\right]\right)= \begin{cases}0, & E\left[c\left(\Delta T_{N}+t\right)\right] \leq E\left[R_{N} t\right], \\ \lambda\left(E\left[c\left(\Delta T_{N}+t\right)\right]-E\left[R_{N} t\right]\right), & E\left[c\left(\Delta T_{N}+t\right)\right]>E\left[R_{N} t\right] .\end{cases}
$$
exists:

According to expression (3), the following equation

$$
\lambda\left(E\left[c\left(\Delta T_{N}+t\right)-R_{N} t\right]\right)=\lambda E\left[L_{N}\right]
$$

Therefore, expression (12) is turned into the following expression:

$$
\begin{aligned}
& \min _{N \in N^{+}} E\left[Y_{N}\right]=\min _{N \in N^{+}}\left(E\left[N E_{D}+P t\right]\right. \\
& \left.\quad-\zeta E\left[c\left(\Delta T_{N}+t\right)-L_{N}\right]+\lambda E\left[L_{N}\right]\right) .
\end{aligned}
$$

Remark 2. For energy efficiency $\zeta$, there is $E_{D} /(c T)<\zeta<$ $P t /(c t)$. Assume that the ST stops observing and transmits data at the time $N$. When the amount of data left undelivered $L_{N}$ is 0 , the value of energy efficiency is minimal. The minimum value is $\left(N E_{D}+P t\right) /(c N T+c t)$. Therefore, energy efficiency $\zeta$ is no smaller than that one. That means $\zeta \geq$ $\left(N E_{D}+P t\right) /(c N T+c t)$ is satisfied.

As $t \leq T$ holds, $\zeta>\left(N E_{D}+P t\right) /(c N T+c T)$ is satisfied.

That is, $\zeta>E_{D} /(c T)+\left(P t-E_{D}\right) /((N+1) c T)$.

Because transmission energy consumption $P t$ is greater than detection energy consumption $E_{D}$, there is $\zeta>E_{D} /(c T)$.
Meanwhile, $P t /(c t)$ is the ratio of transmission energy consumption $P t$ to accumulative data $c t$ during duration $t$. If there is $\zeta>P t /(c t)$, ST would deliver data without channel detection and get smaller value of energy efficiency. Consequently, there is $\zeta<P t /(c t)$.

4.3. Solution to Optimal Stopping Problem about Average Energy Consumption Minimization. To solve the above minimization problem of AECPUDT, firstly the existence of optimal stopping rule needs to be proved. Then, description of the optimal stopping strategy is called for. Finally, the solution to the optimal stopping problem is expected. Proposition 3 is presented as follows.

Proposition 3. There is optimal stopping rule in expression (16).

Proof. According to [17], if the problem satisfies the two conditions,

(A1) $E\left[\inf _{n} Y_{n}\right]>-\infty$,

(A2) $\liminf _{n \rightarrow \infty} Y_{n} \geq Y_{\infty}$ a.s., 
the optimal stopping rule exists. Because $\Delta T_{n}$ is equal to $n T$, expression (16) is converted into the following equation:

$$
Y_{n}=n E_{D}+P t-\zeta c(n T+t)+(\lambda+\zeta) L_{n}
$$
t).

Because $L_{n} \geq 0$ is satisfied, there is $Y_{n} \geq n E_{D}+P t-\zeta c(n T+$

$\zeta<P t /(c t)$ holds, so there is $Y_{n}>n E_{D}-\zeta c n T$.

Meanwhile, there is $\zeta>E_{D} /(c T)$. Furthermore, $n T \leq D_{m}$ is satisfied. Thus, $Y_{n}>-\infty$ holds.

Consequently, condition (A1) is satisfied.

If $n \rightarrow \infty, L_{n} \rightarrow c(n T+t)$ holds. And there is $n E_{D} \rightarrow \infty$ and $c(n T+t) \rightarrow \infty$. Consequently, we have the following expression:

$$
\begin{aligned}
& \liminf _{n \rightarrow \infty} Y_{n} \\
& \quad=\liminf _{n \rightarrow \infty}\left(n E_{D}+P t-\zeta c(n T+t)+(\lambda+\zeta) L_{n}\right) \\
& \quad=\liminf _{n \rightarrow \infty}\left(n E_{D}+P t+\lambda c(n T+t)\right)=\infty .
\end{aligned}
$$

As $Y_{\infty}=\infty$, condition (A2) holds.

ST has to detect channel condition every period $T$ and then decides whether the current moment is the optimal moment to stop detecting and deliver data or not. The decision depends on whether the future expected channel condition is better or not. As described in Section 3.3, this is a finite horizon optimal stopping problem. Thus, the expected value can be derived using backward induction, so as to obtain transmission rate threshold at each step that ST stops detecting and transmits data. Therefore, the optimal stopping rule is that ST verifies whether the current transmission rate reaches or exceeds the corresponding threshold or not. If it reaches or exceeds the threshold, ST stops detection and delivers data. Otherwise, ST continues doing detection. When ST continues detection until the delay boundary, it must transmit data unconditionally. In line with [11], the minimal rate of return $W_{n}(\lambda, \zeta)$ at $n$ is as follows:

$$
\begin{aligned}
& W_{n}(\lambda, \zeta)=\min \left(P t+n E_{D}-\zeta c(n T+t)\right. \\
& \left.\quad+(\lambda+\zeta) L_{n}, V_{M-n-1}(\lambda, \zeta)\right), \\
& V_{M-n-1}(\lambda, \zeta)=E\left[W_{n}(\lambda, \zeta) \mid F_{n}\right] \\
& \quad n=1,2, \ldots, M-1 .
\end{aligned}
$$

Here, $F_{n}$ represents that the related sequence values from 1 to $n$ have been obtained through observation. In expression (19), the energy consumption cost $E_{D}$ of ST detecting channel each time is considered. According to the optimal stopping rule, when the rate of return at $n$ is less than or equal to the expected value $V_{M-n-1}(\lambda, \zeta)$, ST will stop detection and transmit data. Hence, the following inequality is obtained:

$$
P t+n E_{D}-\zeta c(n T+t)+(\lambda+\zeta) L_{n} \leq V_{M-n-1}(\lambda, \zeta)
$$

According to the definition of $L_{n}$ in expression (3), when $c(n T+t)>R_{n} t$ is satisfied, the above expression can be turned into the following:

$$
P t+n E_{D}+\lambda c(n T+t)-(\lambda+\zeta) R_{n} t \leq V_{M-n-1}(\lambda, \zeta) .
$$

That is,

$$
R_{n} \geq \frac{P t+n E_{D}+\lambda c(n T+t)-V_{M-n-1}(\lambda, \zeta)}{(\lambda+\zeta) t}
$$

When $c(n T+t) \leq R_{n} t$ holds, expression (20) can be transformed as follows:

$$
P t+n E_{D}-\zeta c(n T+t) \leq V_{M-n-1}(\lambda, \zeta) .
$$

At last, because of maximum delay constraint $D_{m}$ of this system, ST stops detection and transmits data when the total duration of detection in a round is $M \cdot t$. Consequently, the threshold of transmission rate at the time $M$ is 0 .

Above all, we obtain the threshold of transmission rate to stop detection and deliver data at $n$, which is given in the following expression:

$$
\begin{aligned}
& R_{\mathrm{th}, n}(\lambda, \zeta) \\
& = \begin{cases}\alpha, \quad \beta>\alpha, n=1,2, \ldots, M-1, \\
\beta, & \beta<\alpha, c 1 \text { holds, } n=1,2, \ldots, M-1, \\
0, & n=M,\end{cases} \\
& \alpha=\frac{P t+n E_{D}+\lambda c(n T+t)-V_{M-n-1}(\lambda, \zeta)}{(\lambda+\zeta) t}, \\
& \beta=\frac{c(n T+t)}{t}, \quad P t+n E_{D}-\zeta c(n T+t) \leq V_{M-n-1}(\lambda, \zeta) .
\end{aligned}
$$

Assume that the probability density of transmission rate is $f_{R}(r)$. The cumulative probability of transmission rate at $M$ is $\int_{0}^{R_{\max }} f_{R}(r) d r$, which is denoted as $F \widetilde{R}$. The corresponding expected value is $\int_{0}^{R_{\max }} r f_{R}(r) d r$ that is denoted as $\widetilde{R}$. If transmission rate $R_{M}$ of a round satisfies $R_{M} \geq c(M T+t) / t$, $L_{M}$ is equal to 0 .

Define $R_{\mathrm{th}}=c(M T+t) / t$. If there is $R_{\mathrm{th}}<R_{\max }$, the cumulative probability of transmission rate which is less than $R_{\text {th }}$ at $M$ is $\int_{0}^{R_{\text {th }}} f_{R}(r) d r$. It is denoted as $F \widehat{R}_{\text {th }}$. The corresponding expected value is $\int_{0}^{R_{\mathrm{th}}} r f_{R}(r) d r$, which is denoted as $\widehat{R}_{\mathrm{th}}$. Thus, when ST transmits data at the time $M$, the expected value of the rate of return $V_{0}(\lambda, \zeta)$ is as follows:

$$
V_{0}(\lambda, \zeta)= \begin{cases}\left(P t+M E_{D}\right) F \widetilde{R}+\lambda c(M T+t) F \widehat{R}_{\mathrm{th}}-(\lambda+\zeta) \widehat{R}_{\mathrm{th}} t-\zeta c(M T+t)\left(F \widetilde{R}-F \widehat{R}_{\mathrm{th}}\right), & R_{\mathrm{th}} \leq R_{\max }, \\ \left(P t+M E_{D}+\lambda c(M T+t)\right) F \widetilde{R}-(\lambda+\zeta) \widetilde{R} t, & R_{\mathrm{th}}>R_{\max } .\end{cases}
$$


According to backward induction, the following equation is obtained that combines with expression (19):

$$
\begin{aligned}
& V_{1}(\lambda, \zeta)=E\left[\operatorname { m i n } \left(P t+(M-1) E_{D}\right.\right. \\
& \left.\left.\quad-\zeta c((M-1) T+t)+(\lambda+\zeta) L_{M-1}, V_{0}(\lambda, \zeta)\right)\right] \\
& \quad=\int_{R_{\mathrm{th}, M-1}(\lambda, \zeta)}^{R_{\max }}\left(P t+(M-1) E_{D}-\zeta c((M-1) T\right. \\
& \left.\quad+t)+(\lambda+\zeta) L_{M-1}\right) f_{R}(r) d r \\
& \quad+\int_{0}^{R_{\mathrm{th}, M-1}(\lambda, \zeta)} V_{0}(\lambda, \zeta) f_{R}(r) d r .
\end{aligned}
$$

Similarly, we obtain $V_{2}, \ldots, V_{M-1}$. Thus, the expected value of the rate of return $V_{M-n}(\lambda, \zeta)$ at $n$ is as follows:

$$
\begin{aligned}
& V_{M-n}(\lambda, \zeta)=E\left[\operatorname { m i n } \left(P t+n E_{D}-\zeta c(n T+t)\right.\right. \\
& \left.\left.+(\lambda+\zeta) L_{n}, V_{M-n-1}(\lambda, \zeta)\right)\right]=\int_{R_{\mathrm{th}, n}(\lambda, \zeta)}^{R_{\max }}\left(P t+n E_{D}\right. \\
& \left.-\zeta c(n T+t)+(\lambda+\zeta) L_{n}\right) f_{R}(r) d r \\
& +\int_{0}^{R_{\mathrm{th}, n}(\lambda, \zeta)} V_{M-n-1}(\lambda, \zeta) f_{R}(r) d r \\
& n=1,2, \ldots, M-1,
\end{aligned}
$$

where $R_{\mathrm{th}, n}(\lambda, \zeta)$ is presented in expression (24). Therefore, the optimal stopping rule for expression (16) is presented as follows:

$$
N\left(\zeta^{*}\right)=\min \left\{M \geq n \geq 1: R_{n} \geq R_{\mathrm{th}, n}\left(\lambda, \zeta^{*}\right)\right\},
$$

where $R_{\mathrm{th}, n}\left(\lambda, \zeta^{*}\right)$ is defined in expression (24). Next, it is needed to calculate the value of $\lambda$ and $\zeta^{*}$. According to [17], the optimal stopping rule has the following optimal equation:

$$
\begin{aligned}
& V^{*}\left(\lambda, \zeta^{*}\right)=E\left[\operatorname { m i n } \left(P t+N E_{D}-\zeta^{*} c(N T+t)\right.\right. \\
& \left.\left.\quad+\left(\lambda+\zeta^{*}\right) L_{N}, V^{*}\left(\lambda, \zeta^{*}\right)+E_{D}\right)\right] .
\end{aligned}
$$

And the optimal solution to the equation is $V^{*}\left(\lambda, \zeta^{*}\right)=$ 0 . Consequently, the optimal equation is converted to the following:

$$
\begin{aligned}
0= & E\left[\operatorname { m i n } \left(P t+N E_{D}-\zeta^{*} c(N T+t)\right.\right. \\
& \left.\left.+\left(\lambda+\zeta^{*}\right) L_{N}, E_{D}\right)\right] .
\end{aligned}
$$

Meanwhile, according to the KKT (Karush-KuhnTucker) conditions, the following expression exists:

$$
\lambda c E[(N T+t)]-\lambda E\left[R_{N} t\right]=0 .
$$

To solve (31) it is needed to obtain the value of $E\left[R_{N}\right]$ and $E[N]$. Next, $E\left[R_{N}\right]$ and $E[N]$ are analyzed. In fact, transmission rate $R$ detected by ST at each period $T$ has the same distribution. Moreover, given a cumulative distribution function $F_{G}(g)$ of the random variable channel gain $G$, it is natural to derive the cumulative distribution function $F_{R}(r)$ of the random variable transmission rate $R$. Assume that the random variable transmission rate at $N$ is $R_{N}$. The cumulative distribution function of the random variable $R_{N}$ is as follows:

$$
\begin{aligned}
& F_{R_{N}}(r)=\operatorname{Pr}\left[R_{N, n} \leq r \mid \text { stop at } n\right] \\
& \quad= \begin{cases}\frac{F_{R}(r)-F_{R}\left(R_{\mathrm{th}, n}\left(\lambda, \zeta^{*}\right)\right)}{1-F_{R}\left(R_{\mathrm{th}, n}\left(\lambda, \zeta^{*}\right)\right)}, & r \geq R_{\mathrm{th}, n}\left(\lambda, \zeta^{*}\right), \\
0, & r<R_{\mathrm{th}, n}\left(\lambda, \zeta^{*}\right) .\end{cases}
\end{aligned}
$$

The probability of ST stopping at $n$ is defined as follows:

$$
\rho_{n}=\left(\prod_{i=1}^{n-1} F_{R}\left(R_{\mathrm{th}, i}\left(\lambda, \zeta^{*}\right)\right)\right)\left(1-F_{R}\left(R_{\mathrm{th}, n}\left(\lambda, \zeta^{*}\right)\right)\right) \text {. }
$$

Hence, the expected value of the random variable transmission rate $R_{N}$ is given as follows:

$$
\begin{aligned}
E & {\left[R_{N}\right]=\sum_{n=1}^{M} E\left[R_{N, n} \mid \text { stop at } n\right] \cdot \rho_{n} } \\
& =\sum_{n=1}^{M}\left(\int_{R_{\mathrm{th}, n}\left(\lambda, \zeta^{*}\right)}^{R_{\max }} \frac{r}{1-F_{R}\left(R_{\mathrm{th}, n}\left(\lambda, \zeta^{*}\right)\right)} d F_{R}(r)\right) \cdot \rho_{n} .
\end{aligned}
$$

The expected value of the random variable stopping time $N$ is presented as follows:

$$
E[N]=\sum_{n=1}^{M} n \rho_{n}
$$

Consequently, the following equations can be reasoned out:

$$
\begin{aligned}
\zeta^{*} & =\frac{P t+E[N] E_{D}}{\left(c(E[N] T+t), E\left[R_{N}\right] T\right)^{+}}, \\
\left(c(E[N] T+t), E\left[R_{N}\right] T\right)^{+} & \\
& = \begin{cases}c(E[N] T+t), & c(E[N] T+t) \leq E\left[R_{N}\right] T, \\
E\left[R_{N}\right] T, & c(E[N] T+t)>E\left[R_{N}\right] T .\end{cases}
\end{aligned}
$$

$E\left[R_{N}\right]$ and $E[N]$ are defined in expressions (34) and (35), respectively. Expression (36) is solved to obtain the value of $\zeta^{*}$ and $\lambda$. Process of solving $\zeta^{*}$ and $\lambda$ is described in detail below.

Step 1. Start: let $k=1$, and initialize $\lambda_{k}, \lambda_{\max }$, and $\lambda_{\Delta}$.

Step 2. If $\lambda_{k} \leq \lambda_{\text {max }}$, initialize $\zeta_{0}$, and carry out Step 3, or else go to Step 8.

Step 3. Solve $V_{0}\left(\lambda_{k}, \zeta_{0}\right)$ according to expression (25). Let $n=$ $M-1$.

Step 4. If $n \geq 1$, carry out Step 5, or else go to Step 6 .

Step 5. Obtain $R_{\mathrm{th}, n}\left(\lambda_{k}, \zeta_{0}\right)$ according to expression (24). Compute $V_{M-n}\left(\lambda_{k}, \zeta_{0}\right)$ according to expression (27). Let $n=$ $n-1$. Return to Step 4 . 
Step 6. Obtain $E\left[R_{N}\right]$ and $E[N]$ according to expressions (34) and (35), respectively. Calculate $\zeta_{\text {new }}$ according to expression (36). If $\left|\zeta_{\text {new }}-\zeta_{0}\right|>\varepsilon$ (the error value predefined), let $\zeta_{0}=\zeta_{\text {new }}$ and return to Step 3, or else let $\zeta_{k}^{*}=\zeta_{\text {new }}$ and carry out Step 7.

Step 7. Let $k=k+1$ and $\lambda_{k}=\lambda_{k-1} * \lambda_{\Delta}$. Return to Step 2 .

Step 8. Select the minimum value from the sequence of $\zeta_{k}^{*}$ as $\zeta^{*}$, and the corresponding $\lambda_{k}$ is saved as $\lambda$.

Given the values of $\lambda_{k}$ and $\zeta_{0}, \zeta_{k}^{*}$ is obtained by Newton iterative method. The iterative method is quadratic convergence to the optimal energy efficiency $\zeta_{k}^{*}$, and the result becomes stable after 3 iterations. To get the optimal $\zeta^{*}$, a series of $\zeta_{k}^{*}$ are found out through a series of $\lambda_{k}$, and then the minimal value is selected out as the optimal $\zeta^{*}$. Here, $\lambda_{k} \geq 0$ is Lagrange multiplier. Its value is determined by the value of $\zeta_{k}^{*}$ and is smaller than that one. The experiment showed that the number of values of $\lambda_{k}$ is no more than 10. According to $\zeta^{*}$ and $\lambda$, the optimal transmission rate threshold value $R_{\mathrm{th}, n}\left(\lambda, \zeta^{*}\right)$ is obtained and presented in expression (24).

This optimal transmission rate threshold $R_{\mathrm{th}, n}\left(\lambda, \zeta^{*}\right)$ gives the transmission rate threshold at which ST would stop at every slot time $n$. It also shows the optimal threshold of transmission rate that ST could obtain the smallest AECPUDT. $R_{\mathrm{th}, n}\left(\lambda, \zeta^{*}\right)$ effectively decides the optimal time of ST transmitting data. ST detects channel every period $T$. If ST discovers that the transmission rate at current slot time $n$ is no smaller than $R_{\mathrm{th}, n}\left(\lambda, \zeta^{*}\right)$, ST would stop detection and send data. On the contrary, ST continues doing detection. When ST detects channel till the delay value $M \cdot t$, it has to deliver data. In accordance with this optimal stopping strategy, ST detects channel and delivers data continuously, thus decreasing the AECPUDT and increasing the average delivery ratio.

\section{Simulation Results and Analysis}

The simulation results are shown in this section, which are obtained by MATLAB simulation tools. The relationships between these five parameters and the value of optimal energy efficiency $\zeta^{*}$ are firstly given, so as to determine their values. The parameters include data generation rate $c$, detection energy consumption $E_{D}$, detection cycle $T$, transmission duration $t$, and maximum transmission delay $D_{m}$, respectively. Then, a comparison is made between the results of our proposed strategy and the ones of other strategies under different parameters. The results include the average energy consumption, the average success delivery ratio, and the average scheduling period.

Wireless channel fading is small scale fading, and the model is generally simulated as Rayleigh or Rician distribution. The channel conditions of ST and RT obey the same probability distribution. ST obtains channel condition through signal transmitted by RT periodically, as described in Section 3.1. According to [18], probability density function (PDF) in Rayleigh distribution is as follows:

$$
f_{G}(g)=\frac{g}{\sigma^{2}} \exp \left(-\frac{g^{2}}{2 \sigma^{2}}\right), \quad g \geq 0 .
$$

TABLE 1: Parameter values in simulation.

\begin{tabular}{lcc}
\hline Parameter & Description & Value \\
\hline$W$ & Bandwidth [MHZ] & 1 \\
$N_{0}$ & Noise power spectral density [W/HZ] & $10^{-6}$ \\
$\sigma^{2}$ & Value related to mean variance of channel & 1 \\
$g$ & gain & \\
$P$ & Channel gain & $0 \sim 4$ \\
$A$ & Transmission power [mW] & 100 \\
\hline
\end{tabular}

Here, $g$ is the channel gain and $\sigma^{2}$ is value related to mean variance of channel gain $g$. Combining with expression (1), $R_{\max }$-normalized cumulative distribution function (CDF) of transmission rate $r$ in Rayleigh distribution is presented as follows:

$$
F_{R}(r)=\frac{\exp \left(-\left(2^{r / W}-1\right)^{2} \cdot\left(N_{0} W\right)^{2} /\left(2 \sigma^{2} P^{2}\right)\right)}{\exp \left(-\left(2^{R_{\max } / W}-1\right)^{2} \cdot\left(N_{0} W\right)^{2} /\left(2 \sigma^{2} P^{2}\right)\right)} .
$$

In accordance with [18], PDF in Rician distribution is as follows:

$$
f_{G}(g)=\frac{g}{\sigma^{2}} \exp \left(-\frac{g^{2}+A^{2}}{2 \sigma^{2}}\right) I_{0}\left(\frac{g A}{\sigma^{2}}\right), \quad g \geq 0 .
$$

Here, $I_{0}(\cdot)$ is a Bessel function of first-class 0 -order correction and $A$ is main signal amplitude peak value. Combining with expression (1), $R_{\max }$-normalized CDF of transmission rate $r$ in Rician distribution is obtained as follows:

$$
F_{R}(r)=\frac{Q_{1}\left(A / \sigma,\left(2^{r / W}-1\right) N_{0} W /(\sigma P)\right)}{Q_{1}\left(A / \sigma,\left(2^{R_{\max } / W}-1\right) N_{0} W /(\sigma P)\right)},
$$

where $Q_{1}(\cdot)$ is the first-class Marcum $Q$-function. The parameter values in simulation are shown in Table 1.

5.1. Effects under Different Parameters on Optimal Energy Efficiency $\zeta^{*}$. The cumulative distribution curve of transmission rate $r$ is shown in Figure 3(a). As can be seen from the figure, the cumulative probability of $r$ less than $3 \times 10^{4}$ bps is 0.02 . That is, the opportunity of link obtaining this rate is smaller. The cumulative probability of $r$ less than $1.6 \times 10^{5} \mathrm{bps}$ is 0.5 in Rayleigh distribution and that of $r$ less than $2 \times 10^{5}$ bps is also 0.5 in Rician distribution. This means that the opportunity of link obtaining rate more than that is less than 0.5 . But the data loss probability at this moment will be greater than 0.5 . Thus, the data generation rate $c$ in Rayleigh distribution is set to be between $3 \times 10^{4}$ and $1.6 \times 10^{5}$ bps. That in Rician distribution is between $3 \times 10^{4}$ and $2 \times 10^{5}$ bps.

As can be seen from expression (36), the optimal energy efficiency $\zeta^{*}$ of the strategy proposed by this paper is closely related to five parameters of ST. The curves of relationships between these parameters and $\zeta^{*}$ are shown in Figures 3(b), 3(c), 3(d), 3(e), 3(f), 3(g), and 3(h).

Here, $\zeta^{*}$ is the ratio of the total energy consumption to the amount of data successfully transmitted. The total energy 


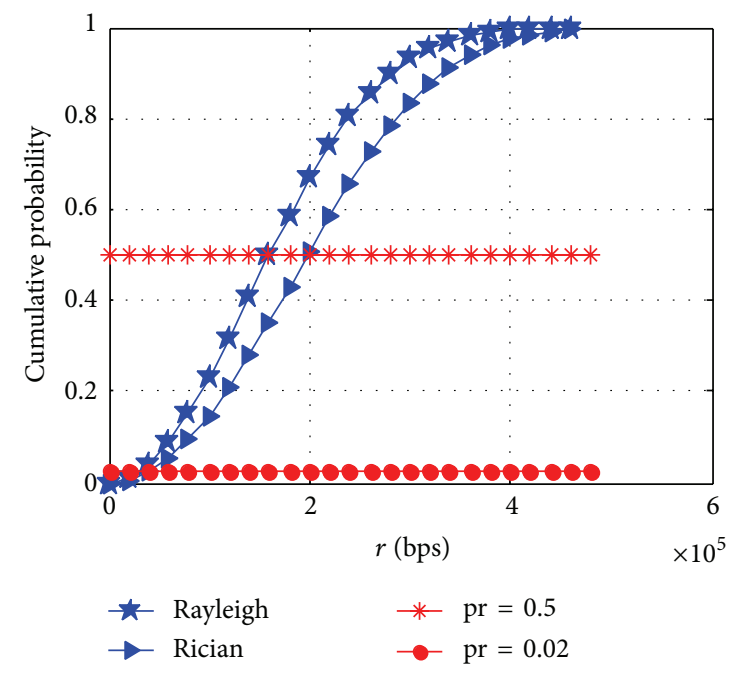

(a) Distribution curve of transmission rate $r$

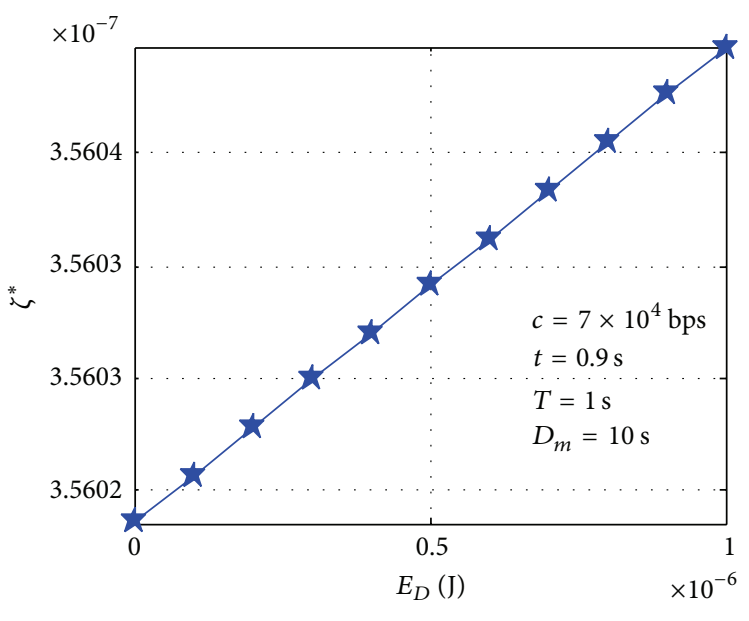

Rayleigh

(c) Relationship of $E_{D}$ and $\zeta^{*}$

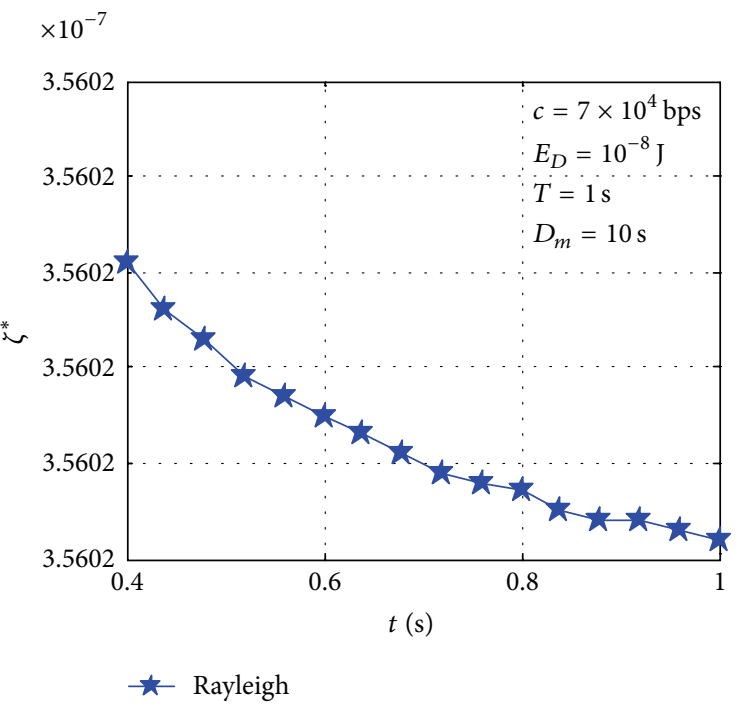

(e) Relationship of $t$ and $\zeta^{*}$

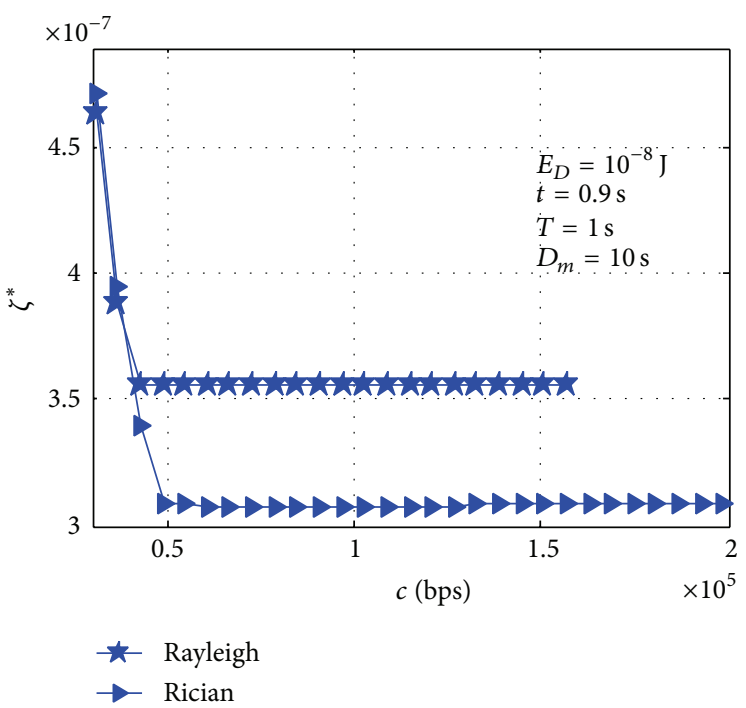

(b) Relationship of $c$ and $\zeta^{*}$

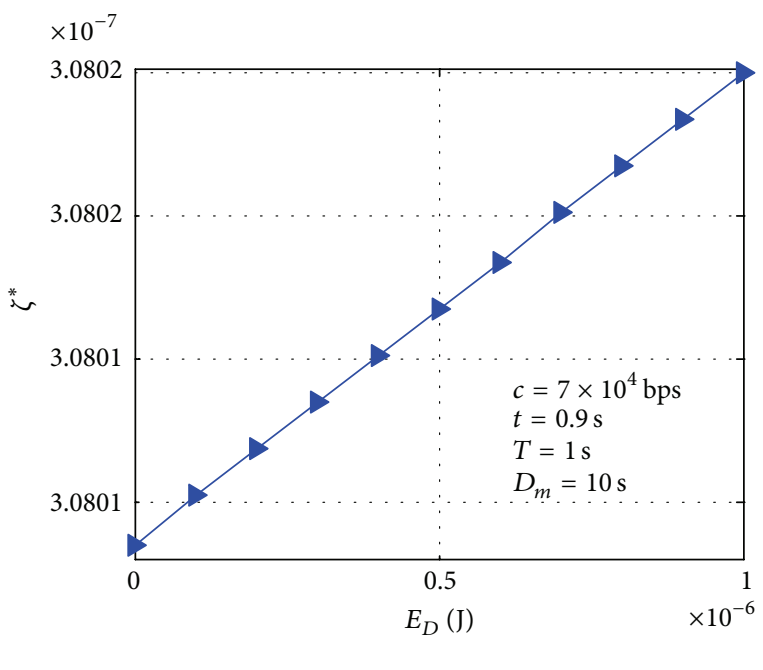

$\rightarrow$ Rician

(d) Relationship of $E_{D}$ and $\zeta^{*}$

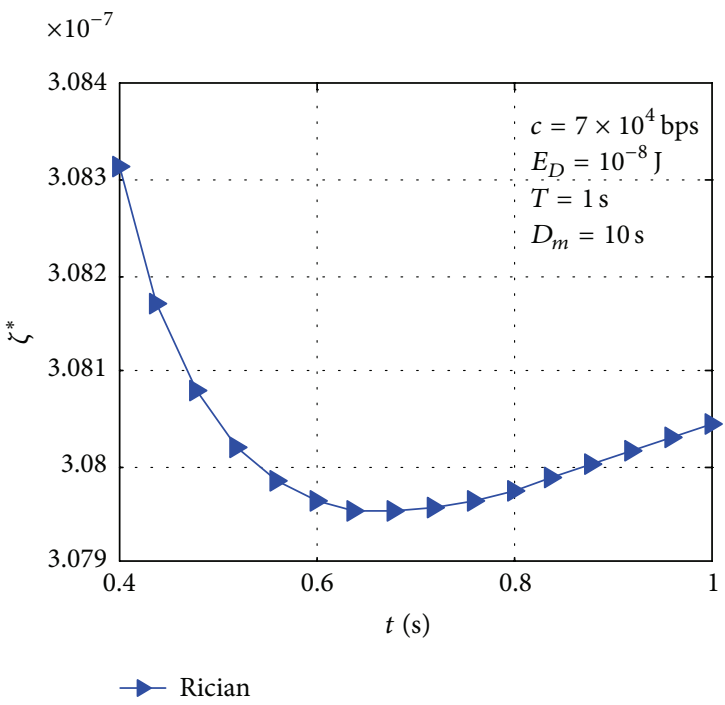

(f) Relationship of $t$ and $\zeta^{*}$

Figure 3: Continued. 


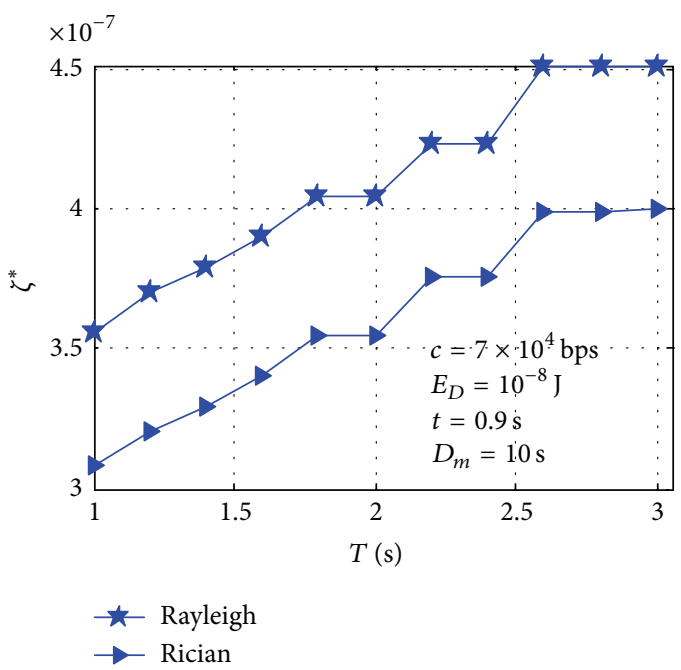

(g) Relationship of $T$ and $\zeta^{*}$

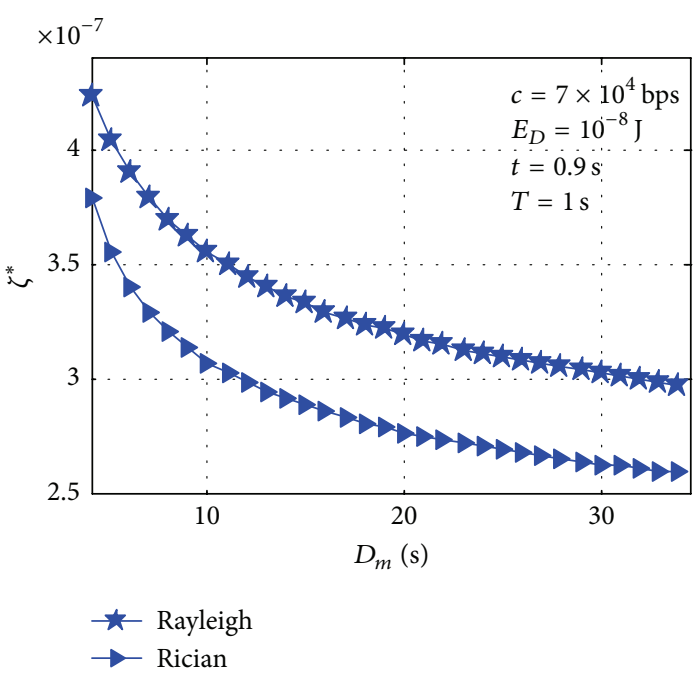

(h) Relationship of $D_{m}$ and $\zeta^{*}$

FIgURE 3: Cumulative distribution curve of transmission rate $r$ and relationships between each parameter and $\zeta^{*}$.

consumption is energy consumed by ST for detection and transmission in every round. Firstly, the energy consumption for transmission in every round is constant. With $c$ decreasing, the amount of data accumulated is reduced. If the amount of data waiting for transmission is less than the one that can be transmitted within $t, \zeta^{*}$ will increase. Conversely, $\zeta^{*}$ will decrease. Secondly, the total energy consumption of ST includes $E_{D}$. Therefore, $\zeta^{*}$ increases with the growth of $E_{D}$ and decreases with the reduction of $E_{D}$. Thirdly, the transmission energy consumption grows with $t$ increasing. If the amount of data accumulated at this moment is less than the one that can be transmitted within $t, \zeta^{*}$ will increase. But the data loss probability reduces. Conversely, $\zeta^{*}$ slightly decreases, but the data loss probability will increase. Fourthly, if $T$ is prolonged, the opportunity that ST finds good channel condition would decrease. So ST has less chance to transmit more data within $t$, and then $\zeta^{*}$ increases. Fifthly, the amount of data accumulated grows with $D_{m}$ increasing. So ST can accumulate more data, before it obtains good channel condition. Consequently, $\zeta^{*}$ decreases, but the data loss probability will increase.

The above analysis shows how the five parameters affect the value of optimal energy efficiency $\zeta^{*}$. In order to balance the optimal energy efficiency and the data loss probability, these values are taken in simulation, respectively, such that $c=7 \times 10^{4} \mathrm{bps}, E_{D}=10^{-8} \mathrm{~J}, T=1 \mathrm{~s}, t=0.9 \mathrm{~s}$, and $D_{m}=10 \mathrm{~s}$.

\subsection{Performance Comparison and Analysis of Different Strate-} gies. In this section, the comparisons will be made between the Energy-Efficient Transmission Strategy based on Optimal Stopping (EETSOS) theory proposed in this paper and the seven strategies of [5]. The analysis will be made to evaluate the results of average energy consumption, average delivery ratio, and average scheduling period. At first, we describe other strategies used for comparison briefly.

(1) Deterministic Transmission Strategy (DTS). ST begins to send data while the time reaches transmission delay $D_{m}$.
(2) Random Transmission Strategy (RTS). From $M$ time of the maximum delay $D_{m}$, ST will select randomly one of them to deliver data in $1 / M$ probability. (3) Probabilistic Transmission Strategy (PTS). ST will deliver data as it forecasts the probability that transmission rate in future being bigger than the present rate would reach or exceed a certain threshold, or else ST goes on doing detection. (4) Average Rate Transmission Strategy (ARTS). ST sends data while the present transmission rate is bigger than the average transmission rate in the past. On the contrary, ST will continue doing detection. (5) Optimal Transmission Strategy Based on Secretary Problem (OTSSP). ST acquires a maximum transmission rate $R_{c \text {-max }}$ in the period of $37 \%$ [5] of the maximum delay $D_{m}$ using channel detection. In the later period of the remaining $63 \%$ of $D_{m}$, when ST discovers some rate is bigger than $R_{c \text {-max }}$, it stops transmitting. (6) Energy-Efficient Opportunistic Transmission Scheduler, E ${ }^{2}$ OTS [5]. This includes E ${ }^{2}$ OTS-I and $E^{2}$ OTS-II, which realize the objective of expected energy consumption minimization and average energy consumption per unit time minimization, respectively. ST will send data as the present power needed is no greater than the optimal threshold of power in corresponding slot time, or else ST will continue doing detection.

5.2.1. Average Energy Consumption. Average energy consumption represents the energy consumption used by transmitting unit data successfully. Its value is the ratio of the total energy consumption to the total amount of data successfully transmitted. The total energy consumption includes not only transmission energy consumption $P t$ but also detection energy consumption $E_{D}$. Comparison results of the average energy consumption of different strategies under different parameters variations of Rayleigh and Rician distribution are shown in Figure 4.

In Figure 4, it shows that EETSOS has the smallest average energy consumption value. It means that EETSOS gains optimal performance of energy consumption optimization. 


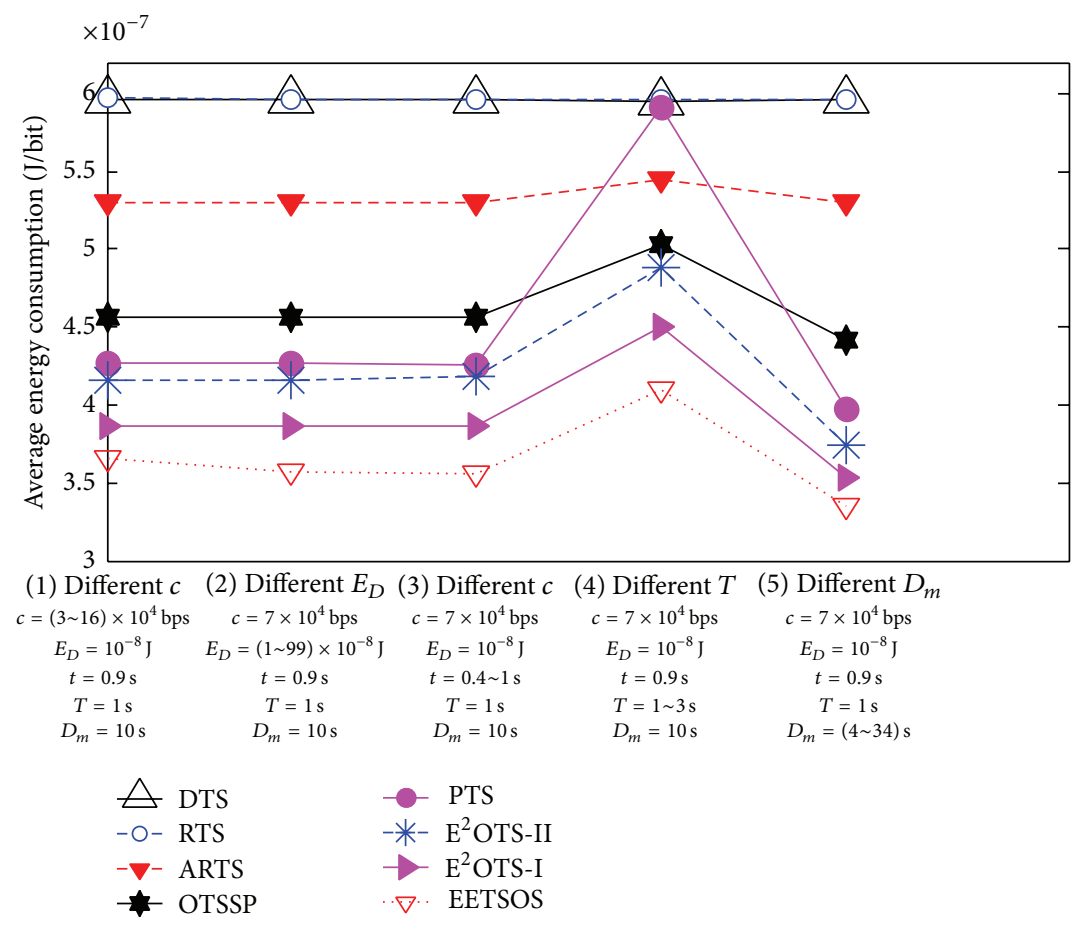

(a) Average energy consumption in Rayleigh distribution

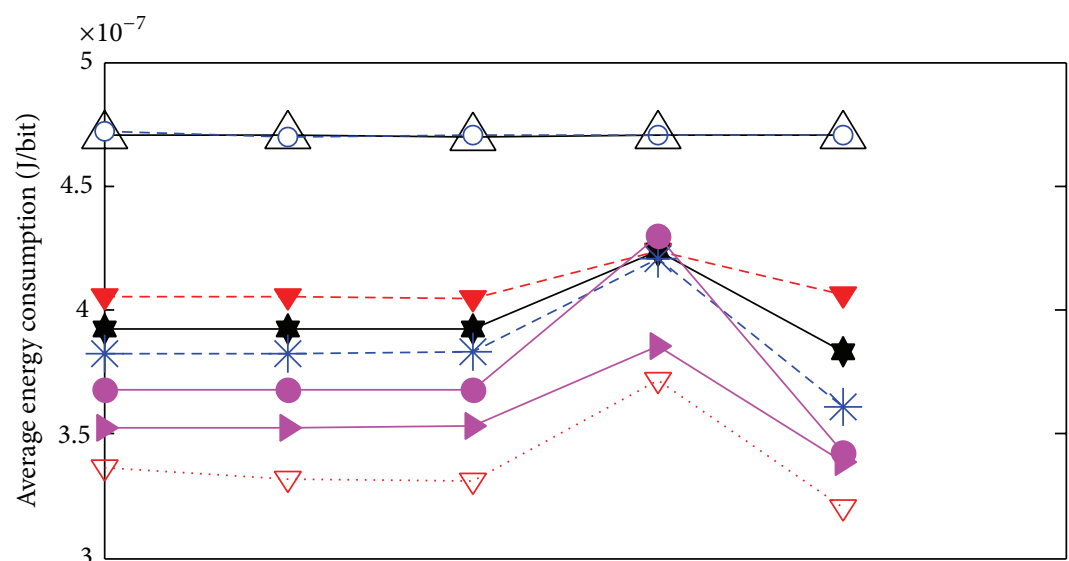

$$
\begin{aligned}
& \text { (1) Different } c \text { (2) Different } E_{D} \text { (3) Different } c \text { (4) Different } T \text { (5) Different } D_{m} \\
& c=(3 \sim 16) \times 10^{4} \mathrm{bps} \quad c=7 \times 10^{4} \mathrm{bps} \quad c=7 \times 10^{4} \mathrm{bps} \quad c=7 \times 10^{4} \mathrm{bps} \quad c=7 \times 10^{4} \mathrm{bps} \\
& E_{D}=10^{-8} \mathrm{~J} \quad E_{D}=(1 \sim 99) \times 10^{-8} \mathrm{~J} \quad E_{D}=10^{-8} \mathrm{~J} \quad E_{D}=10^{-8} \mathrm{~J} \quad E_{D}=10^{-8} \mathrm{~J} \\
& \begin{array}{rlrlrl}
t=0.9 \mathrm{~s} & t=0.9 \mathrm{~s} & t=0.4 \sim 1 \mathrm{~s} & t & =0.9 \mathrm{~s} & t=0.9 \mathrm{~s}
\end{array} \\
& T=1 \mathrm{~s} \quad T=1 \mathrm{~s} \quad T=1 \mathrm{~s} \quad T=1 \sim 3 \mathrm{~s} \quad T=1 \mathrm{~s} \\
& D_{m}=10 \mathrm{~s} \quad D_{m}=10 \mathrm{~s} \quad D_{m}=10 \mathrm{~s} \quad D_{m}=10 \mathrm{~s} \quad D_{m}=(4 \sim 34) \mathrm{s} \\
& \begin{array}{ll}
\triangle \text { DTS } & -*-\text { E}^{2} \text { OTS-II } \\
-0-\text { RTS } & - \text { - PTS } \\
-\nabla \text { - ARTS } & \rightarrow-\text { E}^{2} \text { OTS-I } \\
- \text { - OTSSP } & \therefore \nabla \text { EETSOS }
\end{array}
\end{aligned}
$$

(b) Average energy consumption in Rician distribution

FIGURE 4: Comparisons results of average energy consumption under different parameter variations.

EETSOS gets optimum threshold of transmission rate in every slot time using optimal stopping approach. ST will obtain the exact time of the best energy efficiency to deliver data through comparing the corresponding rate threshold of each slot time, thus decreasing average energy consumption. Since DTS and RTS do not consider energy factor for transmission time selection, they have the biggest average energy consumption values. ARTS, PTS, and OTSSP have much smaller values of average energy consumption compared to RTS and DTS, for they take energy consumption as an important factor for the transmission time selection. However, the three strategies do not obtain a transmission 
time of the best channel quality. So they consume much more average energy than EETSOS and also more one than $\mathrm{E}^{2} \mathrm{OTS}$. $E^{2} O T S$ obtains the smaller average energy consumption value. The average energy consumption value of $\mathrm{E}^{2} \mathrm{OTS}-\mathrm{I}$ is lower than that of $\mathrm{E}^{2} \mathrm{OTS}-\mathrm{II}$. The reason lies in that the objective of $E^{2} \mathrm{OTS}-\mathrm{II}$ is to minimize the average energy consumption per unit time.

5.2.2. Average Delivery Ratio. Average delivery ratio is the ratio of the amount of data successfully transmitted to the total amount of data to be transmitted of ST. The larger value of ratio represents the less amount of data discarded. Comparison results of the average delivery ratio of different strategies under different parameters variations of Rayleigh and Rician distribution are shown in Figure 5.

We observe in Figure 5 that the average delivery ratio of EETSOS is larger than that of other strategies. It means that EETSOS discards relatively small amount of data. When the minimization problem of AECPUDT (see expression (6)) in EETSOS is constructed, one constraint condition to finish all accumulative data transmission is considered. The optimum thresholds of transmission rate in EETSOS ensure improvement of data delivery ratio. DTS has the least average delivery ratio. Since DTS always transmits at the delay $D_{m}$, there will be a lot of data being discarded due to exceeding delay. The average delivery ratios of PTS and RTS are greater than DTS. The two strategies randomly select transmission time and obtain a transmission rate associated with probability distribution. The current rate is compared with the mean value of the past to select transmission time in ARTS, so earlier transmission time to send data is always obtained. Hence, ARTS gets more chances to deliver more data successfully. The average delivery ratio of OTSSP, E ${ }^{2}$ OTS-I, and $E^{2}$ OTS-II is small. Because OTSSP takes $37 \%$ of the maximum delay to detect channel at least, quite a lot of data will be discarded due to exceeding delay. $\mathrm{E}^{2} \mathrm{OTS}-\mathrm{I}$ and $\mathrm{E}^{2} \mathrm{OTS}-\mathrm{II}$ only consider the objective of saving energy and never consider factor of increasing delivery ratio.

5.2.3. Average Scheduling Period. Average scheduling period is the mean value of detection duration of each round in the given simulation time period. The larger this value is, the longer the average detection duration is and, conversely, the shorter it is. Actually, the average scheduling period is the real average delay of data transmission. Comparison results of the average scheduling period of different strategies under different parameter variations of Rayleigh and Rician distribution are shown in Figure 6.

In Figure 6, it shows that the average scheduling period of EETSOS is smaller than that of RTS, DTS, OTSSP, E OTS-I, and $\mathrm{E}^{2} \mathrm{OTS}$-II but is greater than that of RTS, PTS, and ARTS. The average scheduling period of DTS is the longest and equal to the maximum delay. The average scheduling period of RTS is close to the expected value of all detection periods within the maximum delay. The distribution of stopping time of ARTS and PTS is related to the probability distribution of channel. The average scheduling period of OTSSP is longer than $37 \%$ of the maximum delay $D_{m}$. The average scheduling period of $E^{2}$ OTS is related to the power threshold and is longer than that of RTS, PTS, and ARTS. Generally, the longer the average scheduling period is, the larger the amount of data accumulated will be. The probability of data discarded due to exceeding delay is increased. Hence, the average delivery ratio of DTS, RTS, and OTSSP is smaller. EETSOS gets the optimal thresholds of transmission rate with the constraint condition about finishing all accumulative data transmission. Therefore, EETSOS not only reduces the average energy consumption but also increases the delivery ratio.

In summary, the energy-efficient transmission strategy proposed using optimal stopping approach achieves the objective of reducing average energy consumption. In addition, the data delivery ratio under the transmission delay constraint is guaranteed. It means that the strategy of this paper improves energy efficiency under the premise of optimizing performance in mobile network.

\section{Conclusion}

With the rapid advance of network technology, network energy consumption also shows a rapid growth trend. It is one of the important research subjects to construct green mobile computing in the construction and development of mobile networks. In mobile networks, the channel quality in the wireless link varies with time randomly, and it is unstable. If some ST needs to transmit data to another RT in transmission range, ST has to know channel condition timely. In accordance with the information surveyed, ST selects the optimal transmission time to improve energy utilization ratio. Due to the transmission delay demand, it must optimize the data delivery success ratio. Therefore, the minimization problem of AECPUDT with the amount of data transmitted constraint is constructed in this paper. Since wireless channel quality varies with time and distributed opportunistic scheduling can improve network performance and energy utilization of network devices, ST is able to select proper time of the best channel status to send data through distributed opportunistic scheduling. The optimal stopping theory is a useful tool to realize this distributed opportunistic scheduling problem. So this paper proposes transmission strategy using optimal stopping approach, which realizes the objective of decreasing energy consumption through sending data in good channel condition. At first, a minimization problem of AECPUDT is constructed, which considers the transmission delay demand and the amount of data transmitted constraint. This is a finite horizon optimal stopping rule problem. Then the existence of the optimal stopping rule is proved and the solutions and processes of the problem are presented, thus obtaining the optimal threshold of transmission rate on every slot time. ST only compares the current transmission rate obtained by periodical signal with the corresponding optimal threshold and decides which time is the best energy efficiency one to transmit data. Consequently, AECPUDT is reduced, and data delivery ratio is enhanced. The comparison results in simulation indicate that energy-efficient transmission strategy gets the smaller average energy consumption and the larger delivery ratio. The strategy achieves a better optimization 


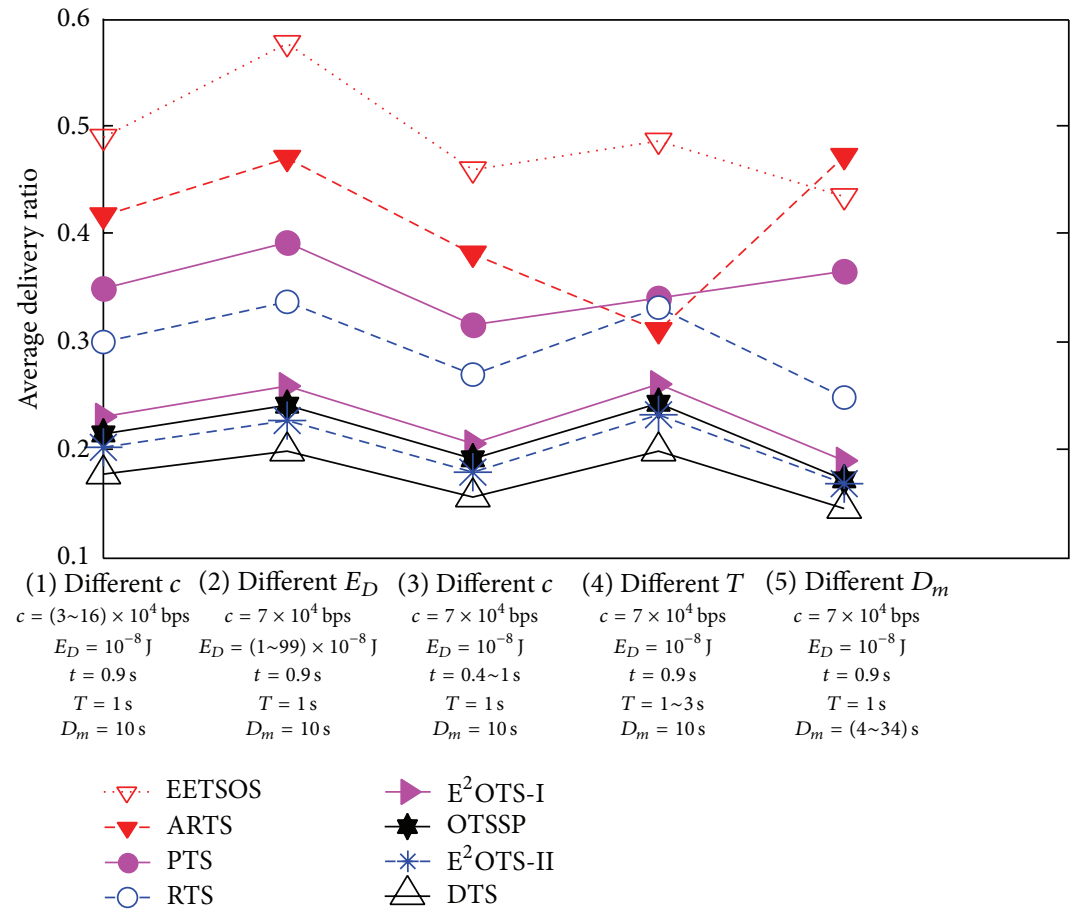

(a) Average delivery ratio in Rayleigh distribution

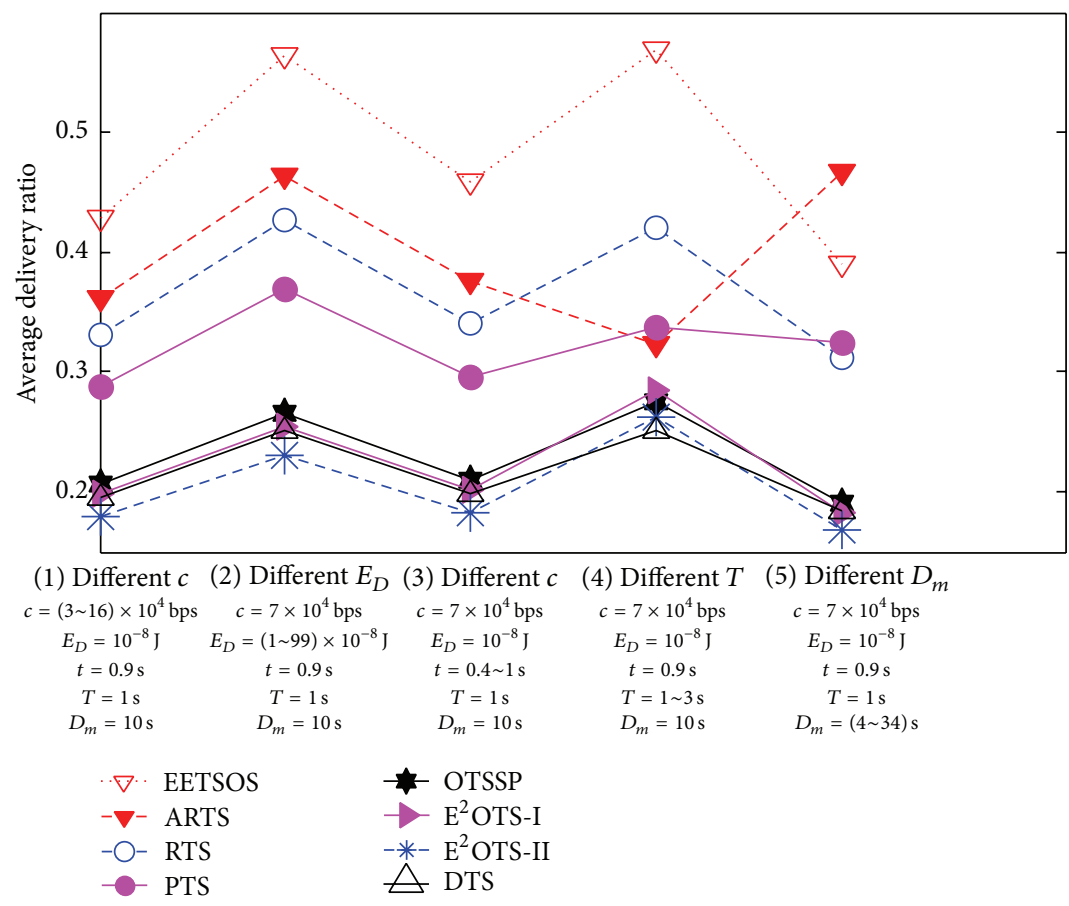

(b) Average delivery ratio in Rician distribution

FIGURE 5: Comparison results of average delivery ratio under different parameter variations. 


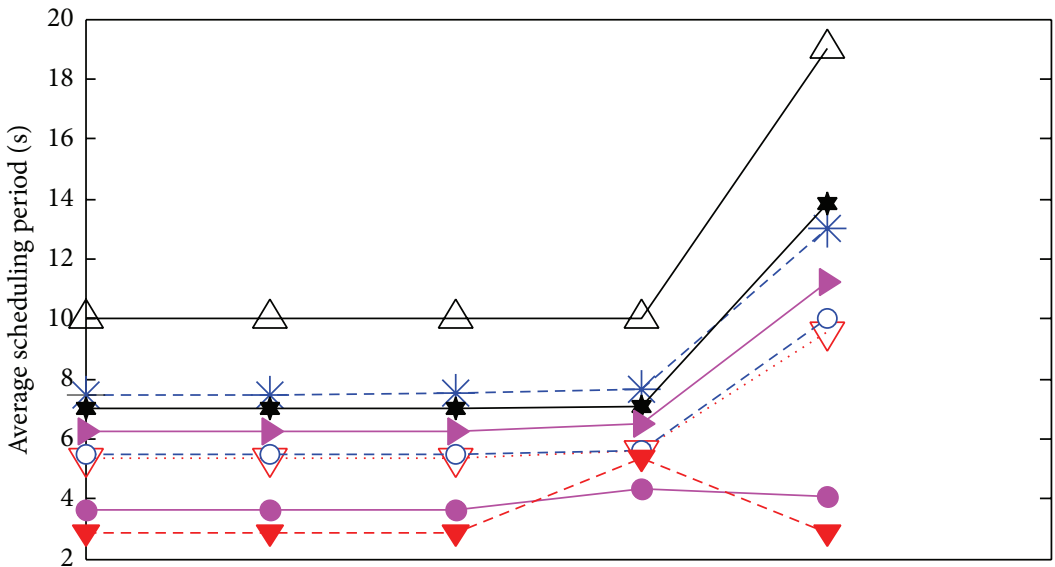

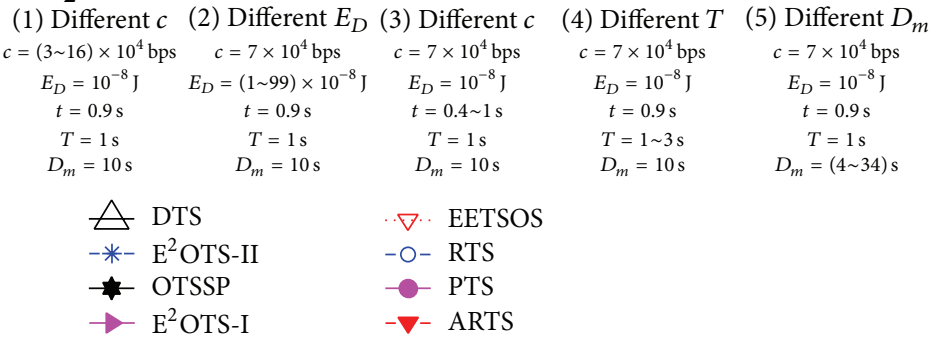

(a) Average scheduling period in Rayleigh distribution

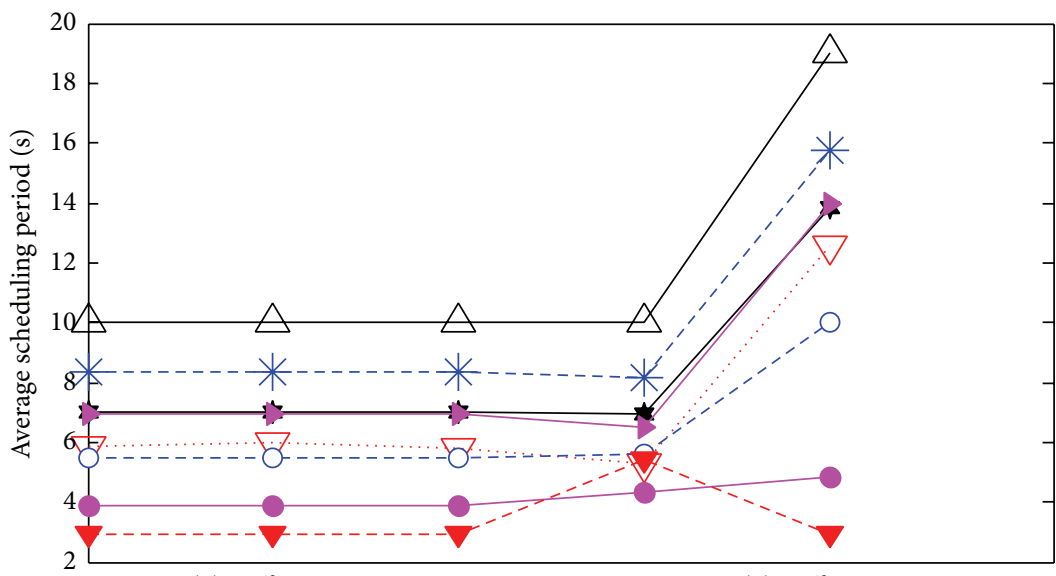

(1) Different $c$ (2) Different $E_{D}$ (3) Different $c$ (4) Different $T$ (5) Different $D_{m}$

$$
\begin{aligned}
& c=(3-16) \times 10^{4} \text { (2) Different } E_{D} \text { (3) Different } c \\
& E_{D}=(1 \sim 99) \times 10^{-8} \mathrm{~J} \quad E_{D}=10^{-8} \\
& t=0.9 \mathrm{~s} \quad t=0.9 \mathrm{~s} \\
& \begin{aligned}
T & =1 \mathrm{~s} \\
D_{m} & =10 \mathrm{~s}
\end{aligned} \\
& T=1 \mathrm{~s} \\
& t=0.4 \sim 1 \mathrm{~s} \\
& D_{m}=10 \mathrm{~s} \\
& T=1 \mathrm{~s} \\
& \begin{array}{ll}
\triangle \text { DTS } & \nabla \text { EETSOS } \\
-*-\text { E}^{2} \text { OTS-II } & -O-\text { RTS } \\
\rightarrow-\text { OTSSP } & -- \text { PTS } \\
\rightarrow \text { E }^{2} \text { OTS-I } & -\nabla-\text { ARTS }
\end{array}
\end{aligned}
$$$$
c=7 \times 10^{4} \mathrm{bps}
$$$$
c=7 \times 10^{4} \mathrm{bps}
$$$$
E_{D}=10^{-8} \mathrm{~J}
$$$$
E_{D}=10^{-8}
$$$$
t=0.9
$$$$
T=1 \sim 3 \mathrm{~s}
$$$$
t=0.9 \mathrm{~s}
$$$$
T=1 \mathrm{~s}
$$

(b) Average scheduling period in Rician distribution

FIGURE 6: Comparison results of average scheduling period under different parameter variations. 
effect of energy consumption on the basis of guaranteeing network performance.

In addition, how to decrease energy consumption and increase delivery ratio in case of changeable data generation rate is a research topic in the future.

\section{Competing Interests}

The authors declare that they have no competing interests.

\section{Acknowledgments}

This research is supported in part by the National Natural Science Foundation of China under Grant nos. 61562006 and 61262003 and in part by the Natural Science Foundation of Guangxi Province under Grant no. 2010GXNSFC013013.

\section{References}

[1] C. Lin, Y. Tian, and M. Yao, "Green network and green evaluation: mechanism, modeling and evaluation," Chinese Journal of Computers, vol. 34, no. 4, pp. 593-612, 2011 (Chinese).

[2] F. Zhang, A. F. Anta, L. Wang, C.-Y. Hou, and Z.-Y. Liu, "Network energy consumption models and energy efficient algorithms," Chinese Journal of Computers, vol. 35, no. 3, pp. 603615, 2012 (Chinese).

[3] A. Asadi and V. Mancuso, "A survey on opportunistic scheduling in wireless communications," IEEE Communications Surveys and Tutorials, vol. 15, no. 4, pp. 1671-1688, 2013.

[4] A. Garcia-Saavedra, P. Serrano, and A. Banchs, "Energy-efficient optimization for distributed opportunistic scheduling," IEEE Communications Letters, vol. 18, no. 6, pp. 1083-1086, 2014.

[5] M. I. Poulakis, A. D. Panagopoulos, and P. Constantinou, "Channel-aware opportunistic transmission scheduling for energy-efficient wireless links," IEEE Transactions on Vehicular Technology, vol. 62, no. 1, pp. 192-204, 2013.

[6] Z. Yan, Z. Zhang, H. Jiang, Z. Shen, and Y. Chang, "Optimal traffic scheduling in vehicular delay tolerant networks," IEEE Communications Letters, vol. 16, no. 1, pp. 50-53, 2012.

[7] L. Huang, H. Jiang, Z. Zhang, and Z. Yan, "Optimal traffic scheduling between roadside units in vehicular delay-tolerant networks," IEEE Transactions on Vehicular Technology, vol. 64, no. 3, pp. 1079-1094, 2015.

[8] P. Ying, N. Wang, and G. Wang, "An optimization strategy of energy consumption for data transmission based on optimal stopping theory in mobile networks," in Algorithms and Architectures for Parallel Processing: 15th International Conference, ICA3PP 2015, Zhangjiajie, China, November 18-20, 2015, Proceedings, Part IV, vol. 9531 of Lecture Notes in Computer Science, pp. 281-292, Springer, Berlin, Germany, 2015.

[9] D. Zheng, W.-Y. Ge, and J.-S. Zhang, "Distributed opportunistic scheduling for ad hoc networks with random access: an optimal stopping approach," IEEE Transactions on Information Theory, vol. 55, no. 1, pp. 205-222, 2009.

[10] A. Garcia-Saavedra, A. Banchs, P. Serrano, and J. Widmer, "Distributed opportunistic scheduling: a control theoretic approach," in Proceedings of the IEEE Conference on Computer Communications (INFOCOM '12), pp. 540-548, IEEE, Orlando, Fla, USA, March 2012.

[11] H. Chen and J. S. Baras, "Distributed opportunistic scheduling for wireless Ad-Hoc networks with block-fading model," IEEE
Journal on Selected Areas in Communications, vol. 31, no. 11, pp. 2324-2337, 2013

[12] W.-G. Mao, S.-S. Wu, and X.-D. Wang, "QoS-oriented distributed opportunistic scheduling for wireless networks with hybrid links," in Proceedings of the IEEE Global Communications Conference (GLOBECOM '13), pp. 4524-4529, Atlanta, Ga, USA, 2013.

[13] C. Van Phan, "A game-theoretic framework for opportunistic transmission in wireless networks," in Proceedings of the 5th IEEE International Conference on Communications and Electronics (ICCE '14), pp. 150-154, Danang, Vietnam, August 2014.

[14] O. Amin and L. Lampe, "Opportunistic energy efficient cooperative communication," IEEE Wireless Communications Letters, vol. 1, no. 5, pp. 412-415, 2012.

[15] J. Zuo, C. Dong, H. V. Nguyen, S. X. Ng, L.-L. Yang, and L. Hanzo, "Cross-layer aided energy-efficient opportunistic routing in ad hoc networks," IEEE Transactions on Communications, vol. 62 , no. 2, pp. 522-535, 2014.

[16] G. Wang, Y. Peng, P. Feng, and N. Wang, "An energy consumption minimization routing scheme based on rate adaptation with QoS guarantee for the mobile environment," Computer Networks, vol. 74, pp. 48-57, 2014.

[17] T. S. Ferguson, "Optimal stopping and applications," 2006, http://www.math.ucla.edu/ tom/Stopping/Contents.html.

[18] S. HayKin, S. Tie-Cheng, X. Ping-Ping et al., Communication System. 4, Publishing House of Electronics Industry, Beijing, China, 2012 (Chinese). 

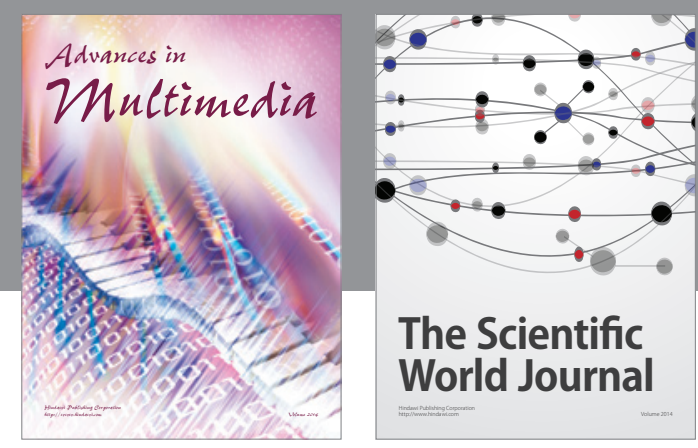

The Scientific World Journal
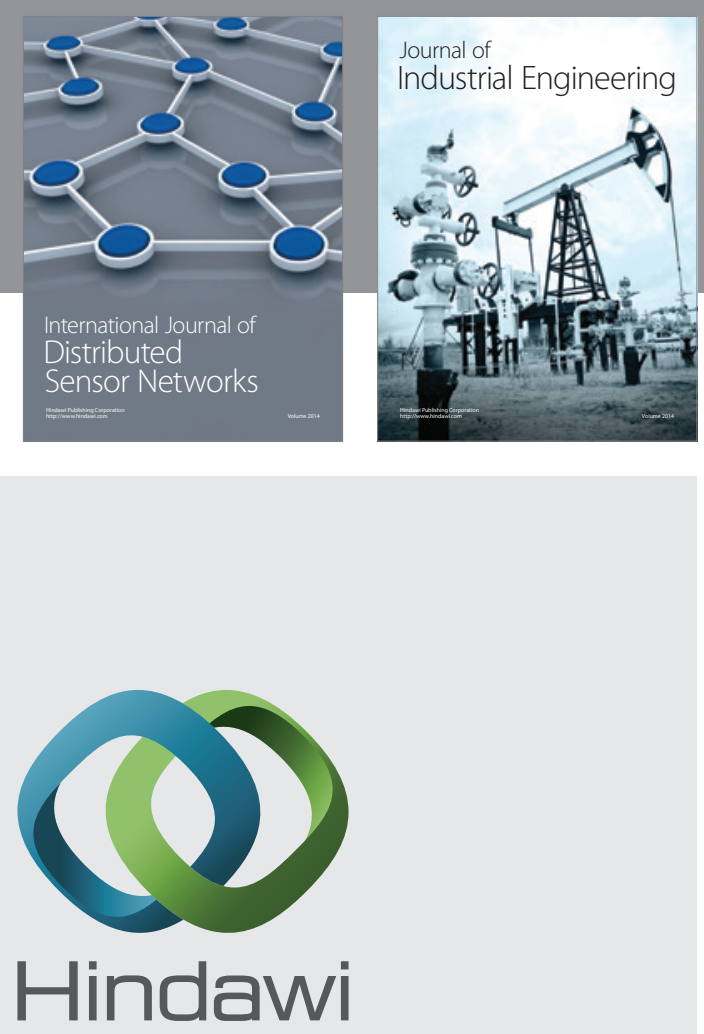

Submit your manuscripts at

http://www.hindawi.com

\section{Computer Networks} and Communications
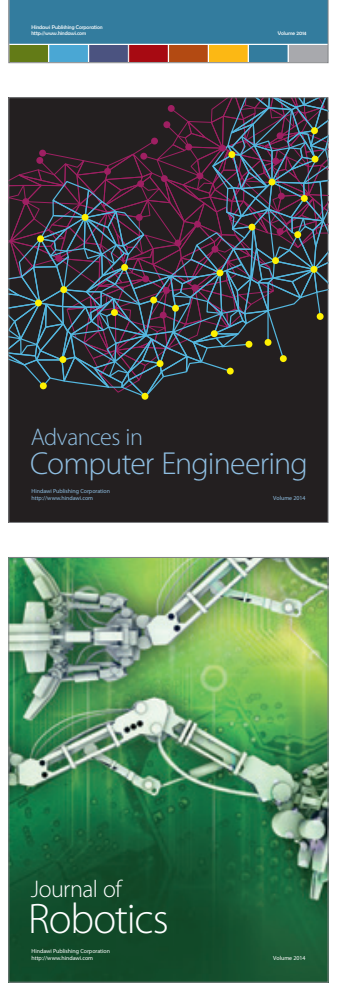
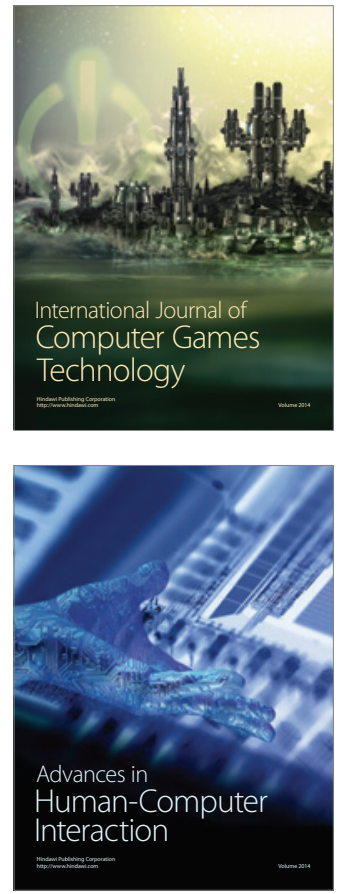
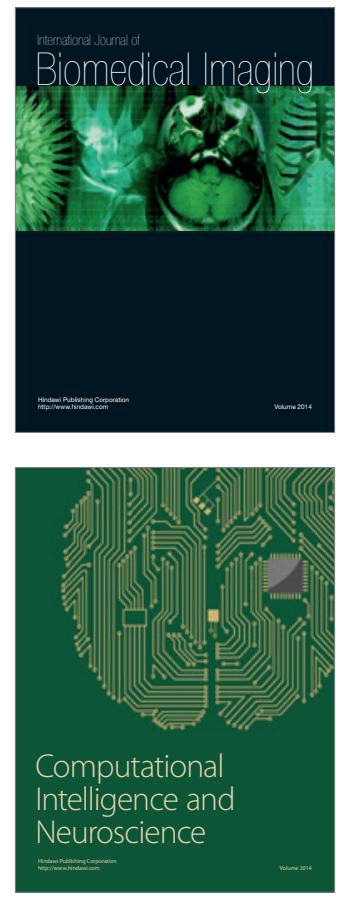
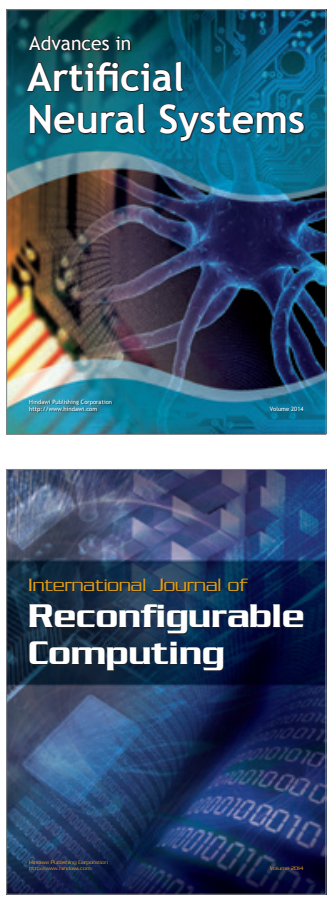
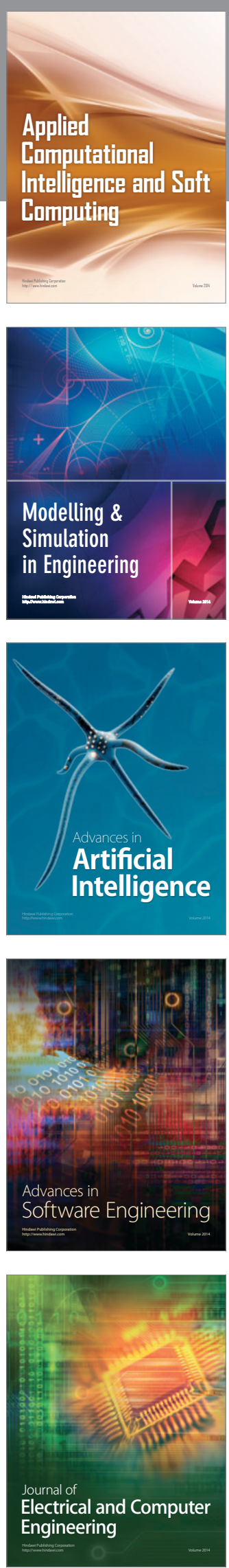